Document downloaded from:

http://hdl.handle.net/10251/107399

This paper must be cited as:

Boiti, C.; Jornet Casanova, D. (2017). A characterization of the wave front set defined by the iterates of an operator with constant coefficients. Revista de la Real Academia de Ciencias Exactas Físicas y Naturales Serie A Matemáticas. 111(3):891-919. doi:10.1007/s13398-0160329-8

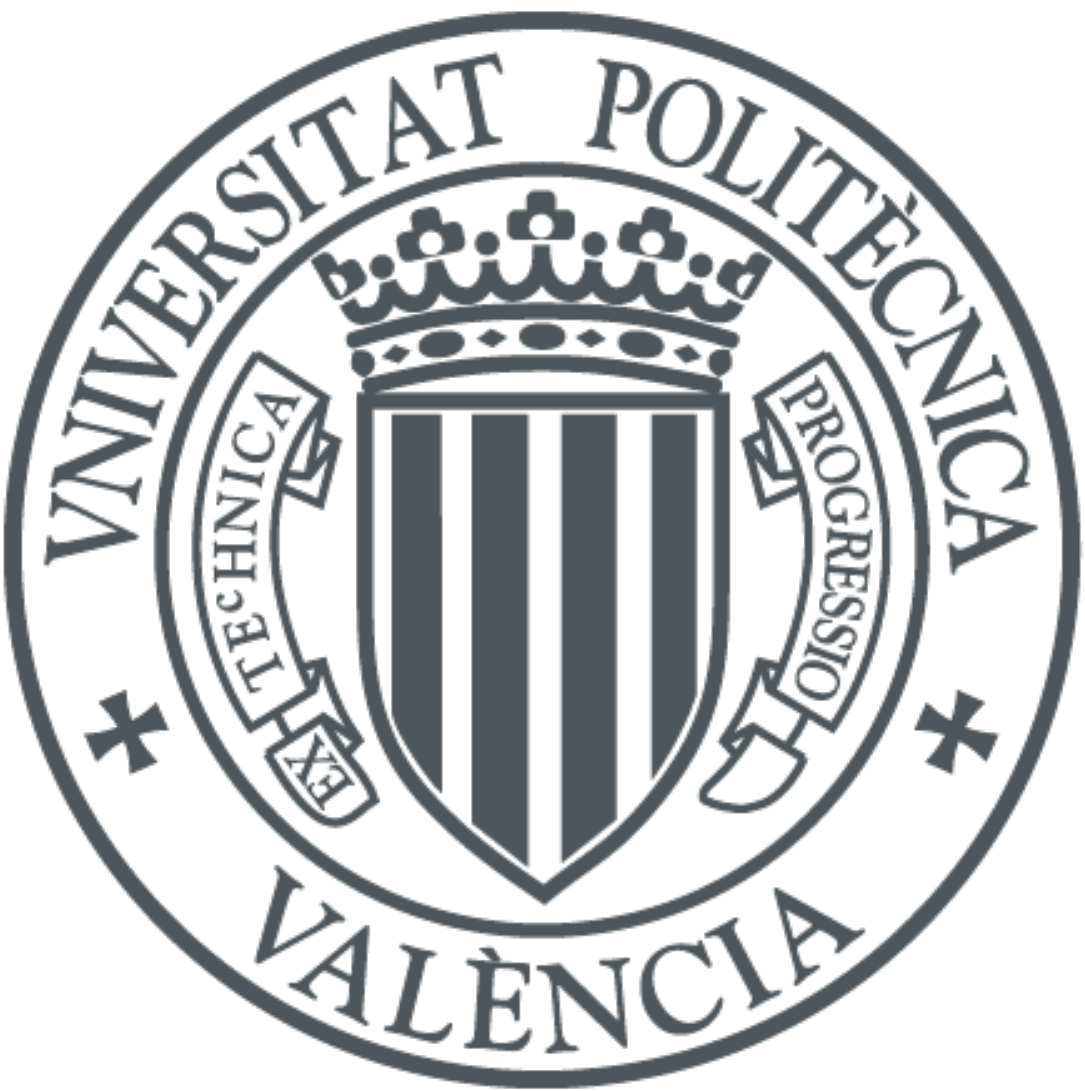

The final publication is available at

http://doi.org/10.1007/s13398-016-0329-8

Copyright Springer-Verlag

Additional Information 


\title{
A CHARACTERIZATION OF THE WAVE FRONT SET DEFINED BY THE ITERATES OF AN OPERATOR WITH CONSTANT COEFFICIENTS
}

\author{
CHIARA BOITI AND DAVID JORNET
}

\begin{abstract}
We characterize the wave front set $\mathrm{WF}_{*}^{P}(u)$ with respect to the iterates of a linear partial differential operator with constant coefficients of a classical distribution $u \in \mathcal{D}^{\prime}(\Omega), \Omega$ an open subset in $\mathbb{R}^{n}$. We use recent Paley-Wiener theorems for generalized ultradifferentiable classes in the sense of Braun, Meise and Taylor. We also give several examples and applications to the regularity of operators with variable coefficients and constant strength. Finally, we construct a distribution with prescribed wave front set of this type.
\end{abstract}

keywords: Iterates of an operator, wave front set, ultradifferentiable functions.

2010 Mathematics Subject Classification: 35A18, 35A20, 35A21.

\section{Introduction}

We introduced in [3] the wave front set $\operatorname{WF}_{*}^{P}(u)$, for a classical distribution $u \in \mathcal{D}^{\prime}(\Omega)$ defined on a open set $\Omega$ of $\mathbb{R}^{n}$, with respect to the iterates of a hypoelliptic linear partial differential operator $P$ with constant coefficients for ultradifferentiable classes in the sense of Braun, Meise and Taylor [7]. We established in [3] a microlocal regularity theorem for this wave front set and we studied the product of ultradifferentiable functions defined in the usual way with the ones defined by iterates. As a consequence we obtained a partial result related to the construction of distributions with prescribed wave front sets. Here, we describe more precisely the behaviour of the set $\operatorname{WF}_{*}^{P}(u)$ and complete the previous result about prescribed singularities. Finally, we give some applications to the $\omega$-microregularity of linear partial differential operators with variable coefficients and constant strength.

The problem of iterates begins mainly when Komatsu [16] in 1960s characterized analytic functions $f$ in terms of the behaviour of successive iterates $P(D)^{j} f$ of a partial differential elliptic operator $P(D)$ with constant coefficients, proving that a $C^{\infty}$ function $f$ is real analytic in $\Omega$ if and only if for every compact set $K \subset \subset \Omega$ there is a constant $C>0$ such that

$$
\left\|P(D)^{j} f\right\|_{2, K} \leq C^{j+1}(j !)^{m},
$$

where $m$ is the order of the operator and $\|\cdot\|_{2, K}$ is the $L^{2}$ norm on $K$. This result was generalized for elliptic operators with variable analytic coefficients by Kotake and Narasimhan [17, Theorem 1]. Later, it was extended to the setting of Gevrey functions by Newberger and Zielezny [23] and completely characterized by Métivier [22] (see also [26]). Spaces of Gevrey type given by the iterates of a differential operator are called generalized Gevrey classes and were used by Langenbruch $[18,19$, $20,21]$ for different purposes. For more references about generalized Gevrey classes and the microlocal version of the problem see [3].

More recently, Juan Huguet [14] extended the results of Komatsu [16], Newberger and Zielezny [23] and Métivier [22] to the setting of non-quasianalytic classes in the sense of Braun, Meise and Taylor [7]. In [14], Juan Huguet introduced the generalized spaces of ultradifferentiable functions $\mathcal{E}_{*}^{P}(\Omega)$ on an open subset $\Omega$ of $\mathbb{R}^{n}$ for a fixed linear partial differential operator $P$ with constant coefficients, and proved that these spaces are complete if and only if $P$ is hypoelliptic. Moreover Juan Huguet showed that, in this case, the spaces are nuclear. Later, the same author in [15] established a Paley-Wiener theorem for the classes $\mathcal{E}_{*}^{P}(\Omega)$, again under the hypothesis of the hypoellipticity of $P$.

In order to remove the assumption on the hypoellipticity of the operator, we considered in [2] a different setting of ultradifferentiable functions, following the ideas of [4]. 
The structure of the paper is as follows. We begin in Section 2 with some notation and preliminaries. First, we introduce the classes of ultradifferentiable functions. Then we complete some partially known results on linear partial differential operators with constant coefficients regarding $\omega$-regularity that we will use in the last section. In Section 3 we use Paley-Wiener theorems in [15] to characterize the wave front set $\mathrm{WF}_{*}^{P}(u)$ introduced in [3] (see Corollaries 3.14 and 3.15). The main tools to establish this characterization are [3, Proposition 17], in which we proved that the product of a suitable Gevrey function and a function in $\mathcal{E}_{*}^{P}(\Omega)$ is still in $\mathcal{E}_{*}^{P}(\Omega)$ (observe that $\mathcal{E}_{*}^{P}(\Omega)$ is not an algebra for pointwise multiplication in general), and the application of pseudodifferential operators defined by symbols supported in a given cone to the description of the wave front set in Theorem 3.11 (see [25, Proposition 3.4.4] for the corresponding result in Gevrey classes). In the last section, Section 4 , we give some applications and examples, in particular to operators with variable coefficients and constant strength. For this purpose we employ some known results on $\omega$-micro-regularity of operators with constant strength (see $[8,9]$ ).

\section{Notation and preliminaries}

Let us recall from [7] the definitions of weight functions $\omega$ and of the spaces of ultradifferentiable functions of Beurling and Roumieu type:

Definition 2.1. A non-quasianalytic weight function is a continuous increasing function $\omega:[0,+\infty[\rightarrow$ $[0,+\infty[$ with the following properties:

$(\alpha) \exists L>0$ s.t. $\omega(2 t) \leq L(\omega(t)+1) \quad \forall t \geq 0$;

(ß) $\int_{1}^{+\infty} \frac{\omega(t)}{t^{2}} d t<+\infty$,

$(\gamma) \log (t)=o(\omega(t))$ as $t \rightarrow+\infty$;

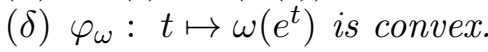

Normally, we will denote $\varphi_{\omega}$ simply by $\varphi$.

For a weight function $\omega$ we define $\bar{\omega}: \mathbb{C}^{n} \rightarrow[0,+\infty[$ by $\bar{\omega}(z):=\omega(|z|)$ and again we denote this function by $\omega$.

The Young conjugate $\varphi^{*}:[0,+\infty[\rightarrow[0,+\infty[$ is defined by

$$
\varphi^{*}(s):=\sup _{t \geq 0}\{s t-\varphi(t)\} .
$$

There is no loss of generality to assume that $\omega$ vanishes on $[0,1]$. Then $\varphi^{*}$ has only non-negative values, it is convex, $\varphi^{*}(t) / t$ is increasing and tends to $\infty$ as $t \rightarrow \infty$, and $\varphi^{* *}=\varphi$.

Example 2.2. The following functions are, after a change in some interval $[0, M]$, examples of weight functions:

(i) $\omega(t)=t^{d}$ for $0<d<1$.

(ii) $\omega(t)=(\log (1+t))^{s}, s>1$.

(iii) $\omega(t)=t(\log (e+t))^{-\beta}, \beta>1$.

(iv) $\omega(t)=\exp \left(\beta(\log (1+t))^{\alpha}\right), 0<\alpha<1$.

In what follows, $\Omega$ denotes an arbitrary subset of $\mathbb{R}^{n}$ and $K \subset \subset \Omega$ means that $K$ is a compact subset in $\Omega$.

Definition 2.3. Let $\omega$ be a weight function.

(a) For a compact subset $K$ in $\mathbb{R}^{n}$ which coincides with the closure of its interior and $\lambda>0$, we define the seminorm

$$
p_{K, \lambda}(f):=\sup _{x \in K} \sup _{\alpha \in \mathbb{N}_{0}^{n}}\left|f^{(\alpha)}(x)\right| \exp \left(-\lambda \varphi^{*}\left(\frac{|\alpha|}{\lambda}\right)\right),
$$

where $\mathbb{N}_{0}:=\mathbb{N} \cup\{0\}$, and set

$$
\mathcal{E}_{\omega}^{\lambda}(K):=\left\{f \in C^{\infty}(K): p_{K, \lambda}(f)<\infty\right\},
$$


which is a Banach space endowed with the $p_{K, \lambda}(\cdot)$-topology. by

(b) For an open subset $\Omega$ in $\mathbb{R}^{n}$, the class of $\omega$-ultradifferentiable functions of Beurling type is defined

$$
\mathcal{E}_{(\omega)}(\Omega):=\left\{f \in C^{\infty}(\Omega): p_{K, \lambda}(f)<\infty, \text { for every } K \subset \subset \Omega \text { and every } \lambda>0\right\} .
$$

The topology of this space is

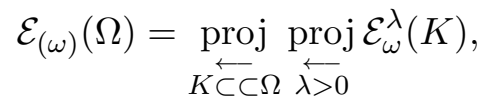

and one can show that $\mathcal{E}_{(\omega)}(\Omega)$ is a Fréchet space.

(c) For a compact subset $K$ in $\mathbb{R}^{n}$ which coincides with the closure of its interior and $\lambda>0$, set

$$
\mathcal{E}_{\{\omega\}}(K)=\left\{f \in C^{\infty}(K) \text { : there exists } m \in \mathbb{N} \text { such that } p_{K, \frac{1}{m}}(f)<\infty\right\},
$$

This space is the strong dual of a nuclear Fréchet space (i.e., a (DFN)-space) if it is endowed with its natural inductive limit topology, that is,

$$
\mathcal{E}_{\{\omega\}}(K)=\underset{\overrightarrow{m \in \mathbb{N}}}{\underset{\operatorname{ind}}{\longrightarrow}} \mathcal{E}_{\omega}^{\frac{1}{m}}(K) .
$$

(d) For an open subset $\Omega$ in $\mathbb{R}^{n}$, the class of $\omega$-ultradifferentiable functions of Roumieu type is defined by:

$$
\mathcal{E}_{\{\omega\}}(\Omega):=\left\{f \in C^{\infty}(\Omega): \forall K \subset \subset \Omega \exists \lambda>0 \text { such that } p_{K, \lambda}(f)<\infty\right\} .
$$

Its topology is the following

$$
\mathcal{E}_{\{\omega\}}(\Omega)=\underset{K \overleftarrow{\text { proj }} \Omega}{\operatorname{\mathcal {E}}} \mathcal{E}_{\{\omega\}}(K)
$$

that is, it is endowed with the topology of the projective limit of the spaces $\mathcal{E}_{\{\omega\}}(K)$ when $K$ runs the compact subsets of $\Omega$. This is a complete PLS-space, that is, a complete space which is a projective limit of LB-spaces (i.e., a countable inductive limit of Banach spaces) with compact linking maps in the (LB)-steps. Moreover, $\mathcal{E}_{\{\omega\}}(\Omega)$ is also a nuclear and reflexive locally convex space. In particular, $\mathcal{E}_{\{\omega\}}(\Omega)$ is an ultrabornological (hence barrelled and bornological) space.

The elements of $\mathcal{E}_{(\omega)}(\Omega)$ (resp. $\mathcal{E}_{\{\omega\}}(\Omega)$ ) are called ultradifferentiable functions of Beurling type (resp. Roumieu type) in $\Omega$.

In the case that $\omega(t):=t^{d}(0<d<1)$, the corresponding Roumieu class is the Gevrey class with exponent $1 / d$. In the limit case $d=1$, not included in our setting, the corresponding Roumieu class $\mathcal{E}_{\{\omega\}}(\Omega)$ is the space of real analytic functions on $\Omega$ whereas the Beurling class $\mathcal{E}_{(\omega)}\left(\mathbb{R}^{n}\right)$ gives the entire functions.

If a statement holds in the Beurling and the Roumieu case then we will use the notation $\mathcal{E}_{*}(\Omega)$. It means that in all cases $*$ can be replaced either by $(\omega)$ or $\{\omega\}$.

For a compact set $K$ in $\mathbb{R}^{n}$, define

$$
\mathcal{D}_{*}(K):=\left\{f \in \mathcal{E}_{*}\left(\mathbb{R}^{n}\right): \operatorname{supp} f \subset K\right\},
$$

endowed with the induced topology. For an open set $\Omega$ in $\mathbb{R}^{n}$, define

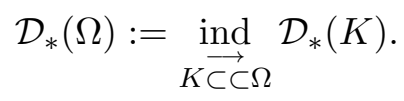

Following [14], we consider smooth functions in an open set $\Omega$ such that there exists $C>0$ verifying for each $j \in \mathbb{N}_{0}:=\mathbb{N} \cup\{0\}$,

$$
\left\|P^{j}(D) f\right\|_{2, K} \leq C \exp \left(\lambda \varphi^{*}\left(\frac{j m}{\lambda}\right)\right),
$$

where $K$ is a compact subset in $\Omega,\|\cdot\|_{2, K}$ denotes the $L^{2}$-norm on $K$ and $P^{j}(D)$ is the $j$-th iterate of the partial differential operator $P(D)$ of order $m$, i.e.,

$$
P^{j}(D)=P(D) \underbrace{0 \cdots \circ}_{j} P(D) \text {. }
$$


If $j=0$, then $P^{0}(D) f=f$.

Given a polynomial $P \in \mathbb{C}\left[z_{1}, \ldots, z_{n}\right]$ of degree $m, P(z)=\sum_{|\alpha| \leq m} a_{\alpha} z^{\alpha}$, the partial differential operator $P(D)$ is defined as $P(D)=\sum_{|\alpha| \leq m} a_{\alpha} D^{\alpha}$, where $D=\frac{1}{i} \partial$.

The spaces of ultradifferentiable functions with respect to the successive iterates of $P$ are defined as follows.

Let $\omega$ be a weight function. Given a polynomial $P$, an open set $\Omega$ of $\mathbb{R}^{n}$, a compact subset $K \subset \subset \Omega$ and $\lambda>0$, we define the seminorm

$$
\|f\|_{K, \lambda}:=\sup _{j \in \mathbb{N}_{0}}\left\|P^{j}(D) f\right\|_{2, K} \exp \left(-\lambda \varphi^{*}\left(\frac{j m}{\lambda}\right)\right)
$$

and set

$$
\mathcal{E}_{P, \omega}^{\lambda}(K)=\left\{f \in \mathcal{C}^{\infty}(K):\|f\|_{K, \lambda}<+\infty\right\} .
$$

It is a normed space endowed with the $\|\cdot\|_{K, \lambda}$-norm.

The space of ultradifferentiable functions of Beurling type with respect to the iterates of $P$ is:

$$
\mathcal{E}_{(\omega)}^{P}(\Omega)=\left\{f \in \mathcal{C}^{\infty}(\Omega):\|f\|_{K, \lambda}<+\infty \text { for each } K \subset \subset \Omega \text { and } \lambda>0\right\},
$$

endowed with the topology given by

$$
\mathcal{E}_{(\omega)}^{P}(\Omega):=\underset{K \subset \subset \Omega}{\operatorname{proj}} \underset{\lambda>0}{\stackrel{\leftarrow}{\operatorname{proj}}} \mathcal{E}_{P, \omega}^{\lambda}(K)
$$

If $\left\{K_{n}\right\}_{n \in \mathbb{N}}$ is a compact exhaustion of $\Omega$ we have

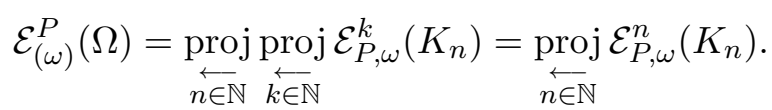

This is a metrizable locally convex topology defined by the fundamental system of seminorms $\left\{\|\cdot\|_{K_{n}, n}\right\}_{n \in \mathbb{N}} \cdot$

The space of ultradifferentiable functions of Roumieu type with respect to the iterates of $P$ is defined by:

$$
\mathcal{E}_{\{\omega\}}^{P}(\Omega)=\left\{f \in \mathcal{C}^{\infty}(\Omega): \forall K \subset \subset \Omega \exists \lambda>0 \text { such that }\|f\|_{K, \lambda}<+\infty\right\} .
$$

Its topology is defined by

$$
\mathcal{E}_{\{\omega\}}^{P}(\Omega):=\underset{K \subset \subset \subset \Omega}{\operatorname{proj}} \underset{\lambda>0}{\operatorname{ind}} \mathcal{E}_{P, \omega}^{\lambda}(K)
$$

As in the Gevrey case, we call these classes generalized non-quasianalytic classes. We observe that in comparison with the spaces of generalized non-quasianalytic classes as defined in [14] we add here $m$ as a factor inside $\varphi^{*}$ in (2.1), where $m$ is the order of the operator $P$, which does not change the properties of the classes and will simplify the notation in the following. In fact, the space $\mathcal{E}_{P, \sigma}(\Omega)$ defined by Juan-Huguet [14], where $\sigma$ is now either $(\sigma)$ or $\{\sigma\}$, satisfies

$$
\mathcal{E}_{P, \sigma}(\Omega)=\mathcal{E}_{\omega}^{P}(\Omega)
$$

for $\sigma(t)=\omega\left(t^{1 / m}\right)$ being $m$ the order of the operator $P$. To be more precise, we have the equality $\varphi_{\sigma}^{*}(x)=\varphi_{\omega}^{*}(m x)($ see $[14$, p. 273$])$.

The inclusion map $\mathcal{E}_{*}(\Omega) \hookrightarrow \mathcal{E}_{*}^{P}(\Omega)$ is continuous (see [14, Theorem 4.1]). The space $\mathcal{E}_{*}^{P}(\Omega)$ is complete if and only if $P$ is hypoelliptic (see [14, Theorem 3.3]). Moreover, under a mild condition on $\omega$ introduced by Bonet, Meise and Melikhov $[6], \mathcal{E}_{*}^{P}(\Omega)$ coincides with the class of ultradifferentiable functions $\mathcal{E}_{*}(\Omega)$ if and only if $P$ is elliptic (see [14, Theorem 4.12]).

We denote by

$$
\widehat{f}(\xi):=\int e^{-i\langle x, \xi\rangle} f(x) d x
$$

the classical Fourier transform $\mathcal{F}(f)$. 
Now, let $P(D)$ be a linear partial differential operator with constant coefficients. We recall the notion of hypoellipticity in the $C^{\infty}$ class: $P(D)$ is hypoelliptic in $\Omega \subseteq \mathbb{R}^{n}$ if $P(D) u \in C^{\infty}(\Omega)$ implies $u \in C^{\infty}(\Omega)$. In this case we also say that the polynomial $P(\xi)=\sum_{|\alpha| \leq m} c_{\alpha} \xi^{\alpha}$ is hypoelliptic.

We set

$$
V=V(P):=\left\{\zeta \in \mathbb{C}^{n}: P(\zeta)=0\right\}
$$

and consider the distance from $\xi \in \mathbb{R}^{n}$ to $V$ :

$$
d(\xi):=\inf _{\zeta \in V}|\xi-\zeta|, \quad \xi \in \mathbb{R}^{n} .
$$

From [13, Thms 11.1.1 and 11.1.3] (see also [25, Prop. 2.2.1]) we recall the following characterization of hypoellipticity, that will be useful in the following:

Theorem 2.4. Let $P(D)$ be a linear partial differential operator with constant coefficients. The following properties are equivalent for $P$ to be hypoelliptic:

(1) For every open set $\Omega \subseteq \mathbb{R}^{n}$ and $u \in \mathcal{D}^{\prime}(\Omega)$

$$
\mathrm{WF}(u)=\mathrm{WF}(P(D) u) .
$$

(2) For every open set $\Omega \subseteq \mathbb{R}^{n}$ and $u \in \mathcal{D}^{\prime}(\Omega)$

$$
\operatorname{sing} \operatorname{supp} u=\operatorname{sing} \operatorname{supp} P(D) u \text {. }
$$

(3) $P$ is homogeneous hypoelliptic, i.e. if $\Omega$ is open in $\mathbb{R}^{n}$ and $u \in \mathcal{D}^{\prime}(\Omega)$ then $P(D) u=0$ implies $u \in C^{\infty}(\Omega)$.

(4) $\lim _{\substack{\xi \in \mathbb{R}^{n} \\|\xi| \rightarrow+\infty}} \frac{D^{\alpha} P(\xi)}{P(\xi)}=0 \quad \forall \alpha \neq 0$.

(5) $P(D)$ has a fundamental solution $E$ with $\operatorname{sing} \operatorname{supp} E=\{0\}$.

(6) $\lim _{\substack{\xi \in \mathbb{R}^{n} \\|\xi| \rightarrow+\infty}} d(\xi)=+\infty$.

(7) $\lim _{\substack{\zeta \in V \\|\zeta| \rightarrow+\infty}}|\operatorname{Im} \zeta|=+\infty$.

(8) There exist $C>0$ and a largest $0<c \leq 1$, with $c \in \mathbb{Q}$, such that for all $\alpha \neq 0$ :

$$
\left|\frac{D^{\alpha} P(\xi)}{P(\xi)}\right| \leq C|\xi|^{-|\alpha| c} \quad \xi \in \mathbb{R}^{n},|\xi| \gg 1 .
$$

(9) There exist $C>0$ and a largest $0<c \leq 1$, with $c \in \mathbb{Q}$, such that

$$
d(\xi) \geq C|\xi|^{c}, \quad \xi \in \mathbb{R}^{n},|\xi| \gg 1 .
$$

Here $\operatorname{WF}(u)$ and sing supp $u$ denote the classical wave front set and singular support of $u \in \mathcal{D}^{\prime}(\Omega)$, as defined in [12].

Remark 2.5. By [13, Lemma 11.1.4] there exists a constant $C>0$ such that for all polynomials $P$ of degree $\leq m$

$$
C^{-1} \leq d(\xi) \sum_{\alpha \neq 0}\left|\frac{D^{\alpha} P(\xi)}{P(\xi)}\right|^{\frac{1}{|\alpha|}} \leq C \quad \text { for } \xi \in \mathbb{R}^{n} \text { with } P(\xi) \neq 0 .
$$

Therefore the constant $c$ at the exponent in (8) and (9) of Theorem 2.4 coincide.

In particular, from (8) with $|\alpha|=m$ we have that if $P$ is hypoelliptic and of order $m$ then there exist $\delta, d>0$ such that

$$
|P(\xi)| \geq \delta|\xi|^{d} \quad \xi \in \mathbb{R}^{n},|\xi| \gg 1
$$

with $m c=d \leq m$.

Moreover, by [10, Thm 3.1], there exists a smallest constant $\gamma:=\gamma(P)>0$, which depends on $P$ and will be relevant later such that

$$
\left|D^{\alpha} P(\xi)\right|^{2} \leq C\left(1+|P(\xi)|^{2}\right)^{1-\frac{|\alpha|}{\gamma}} \quad \forall \xi \in \mathbb{R}^{n} \quad \forall \alpha \in \mathbb{N}_{0}^{n},
$$


for some $C>0$. Note that $m \leq \gamma \leq m / c$, since $b:=1 / \gamma$ has been determined in Theorem 3.1 of [10] applying the Tarski-Seidenberg theorem to

$$
M(\lambda):=\sup _{|P(\xi)|=\lambda}|\operatorname{grad} P(\xi)|=A \lambda^{1-b}(1+o(1)),
$$

and (8) of Theorem 2.4 implies

$$
M(\lambda) \leq A^{\prime} \lambda^{1-\frac{c}{m}}, \quad \lambda \gg 1
$$

for some $A, A^{\prime}>0$, if $P$ has order $m$; (2.6) and (2.7) imply $b \geq c / m$ and hence $m \leq \gamma \leq m / c(\gamma \geq m$ by (2.5) with $|\alpha|=m)$.

We want to generalize Theorem 2.4 to $\omega$-hypoellipticity, for a weight function $\omega$.

Definition 2.6. A linear partial differential operator $P(D)$ with constant coefficients is said to be $(\omega)$-hypoelliptic $\left(\{\omega\}\right.$-hypoellpitic) if every solution $u \in \mathcal{D}^{\prime}\left(\mathbb{R}^{n}\right)$ of $P(D) u=f$ is in $\mathcal{E}_{(\omega)}\left(\mathbb{R}^{n}\right)$ whenever $f \in \mathcal{E}_{(\omega)}\left(\mathbb{R}^{n}\right)\left(u \in \mathcal{E}_{\{\omega\}}\left(\mathbb{R}^{n}\right)\right.$ whenever $\left.f \in \mathcal{E}_{\{\omega\}}\left(\mathbb{R}^{n}\right)\right)$.

We have the following characterization of ( $\omega)$-hypoellipticity (Beurling case):

Theorem 2.7. Let $P(D)$ be a linear partial differential operator with constant coefficients and $\omega$ a non-quasianalytic weight function. The following conditions are equivalent:

(1) $P(D)$ is $(\omega)$-hypoelliptic.

(2) $\lim _{\substack{\zeta \in V \\|\zeta| \rightarrow+\infty}} \frac{|\operatorname{Im} \zeta|}{\omega(\zeta)}=+\infty$

(3) $\lim _{\substack{\xi \in \mathbb{R}^{n} \\|\xi| \rightarrow+\infty}} \frac{\omega(\xi)}{d(\xi)}=0$.

(4) $\lim _{\substack{\xi \in \mathbb{R}^{n} \\|\xi| \rightarrow+\infty}} \frac{\omega(\xi)^{|\alpha|}\left|D^{\alpha} P(\xi)\right|}{|P(\xi)|}=0 \quad \forall \alpha \neq 0$.

Proof. $(1) \Leftrightarrow(2)$ is proved in [5].

$(3) \Rightarrow(2)$ : If $\zeta \in V$ and $|\operatorname{Im} \zeta| \leq|\operatorname{Re} \zeta|$, then from property $(\alpha)$ of $\omega$ we have that

$$
\frac{|\operatorname{Im} \zeta|}{\omega(\zeta)} \geq \frac{d(\operatorname{Re} \zeta)}{L(\omega(\operatorname{Re} \zeta)+1)} \longrightarrow+\infty
$$

by $(3)$.

If $\zeta \in V$ and $|\operatorname{Re} \zeta| \leq|\operatorname{Im} \zeta|$ then

$$
\frac{|\operatorname{Im} \zeta|}{\omega(\zeta)} \geq \frac{1}{2} \frac{|\operatorname{Re} \zeta|+|\operatorname{Im} \zeta|}{\omega(\zeta)} \longrightarrow+\infty
$$

since $\omega(t)=o(t)$.

$(2) \Rightarrow(3)$ : For every fixed $\xi \in \mathbb{R}^{n}$ take $\zeta \in V$ with $|\zeta-\xi| \leq 2 d(\xi)$. Take $|\xi|$ large enough.

If $|\zeta-\xi| \leq|\zeta|$, then $|\xi| \leq 2|\zeta|$ and

$$
\frac{d(\xi)}{\omega(\xi)} \geq \frac{1}{2} \frac{|\zeta-\xi|}{\omega(\xi)} \geq \frac{1}{2} \frac{|\operatorname{Im} \zeta|}{L(\omega(\zeta)+1)} \longrightarrow+\infty
$$

because of (2).

In $|\zeta-\xi| \geq|\zeta|$, then (3) follows from the inequality

$$
\frac{d(\xi)}{\omega(\xi)} \geq \frac{1}{2} \max \left\{\frac{|\xi|}{\omega(\xi)}-\frac{|\zeta|}{\omega(\xi)}, \frac{|\zeta|}{\omega(\xi)}\right\},
$$

since $\omega(t)=o(t)$.

(3) $\Leftrightarrow(4)$ : follows from (2.3). 
Example 2.8. Theorem 2.7 shows, for example, that the heat operator $P=\partial_{t}-\Delta_{x}$ is not $\left(t^{1 / 2}\right)$ hypoelliptic, since

$$
\frac{|(\tau, \xi)|^{2 a}\left|D_{x_{j}}^{2} P(\tau, \xi)\right|}{|P(\tau, \xi)|}=\frac{\left(\tau^{2}+|\xi|^{2}\right)^{a} \cdot 2}{\left.|i \tau+| \xi\right|^{2} \mid}=\frac{2\left(\tau^{2}+|\xi|^{2}\right)^{a}}{\sqrt{\tau^{2}+|\xi|^{4}}} \nrightarrow 0
$$

for $\tau=|\xi| \rightarrow+\infty$ if $2 a \geq 1$. On the other hand, it is well known that the heat operator is $\left\{t^{1 / 2}\right\}$ hypoelliptic (as can be seen also by Theorem 2.9 below).

In the Roumieu case we have the following theorem of characterization of $\{\omega\}$-hypoellipticity, which generalizes Proposition 2.2.1 of [25] (where a similar result is given for the Gevrey classes):

Theorem 2.9. Let $P(D)$ be a linear partial differential operator with constant coefficients and $\omega$ a non-quasianalytic weight function. The following conditions are equivalent:

(1) $P(D)$ is $\{\omega\}$-hypoelliptic.

(2) $\liminf _{\zeta \in V,|\zeta| \rightarrow+\infty} \frac{|\operatorname{Im} \zeta|}{\omega(\zeta)}>0$.

(3) There exists $c>0$ such that

$$
\omega(\zeta) \leq c(1+|\operatorname{Im} \zeta|) \quad \forall \zeta \in V .
$$

(4) There exist $c, C>0$ such that

$$
\omega(\xi) \leq C d(\xi) \quad \text { for } \xi \in \mathbb{R}^{n},|\xi|>c .
$$

(5) There exist $c, C>0$ such that

$$
\left|D^{\alpha} P(\xi)\right| \leq C|P(\xi)| \omega(\xi)^{-|\alpha|} \quad \text { for } \xi \in \mathbb{R}^{n},|\xi|>c, \alpha \in \mathbb{N}_{0}^{n} .
$$

Proof. (1) $\Leftrightarrow(2)$ is proved in [5].

$(2) \Leftrightarrow(3)$ : it is easy to check.

$(3) \Rightarrow(4)$ : For every fixed $\xi \in \mathbb{R}^{n}$ take $\zeta \in V$ with $|\zeta-\xi| \leq 2 d(\xi)$.

If $|\zeta| \leq|\xi-\zeta|$ then $|\xi| \leq 2|\xi-\zeta|$ and for $|\xi|$ large enough

$$
\omega(\xi) \leq \omega(2|\xi-\zeta|) \leq c|\xi-\zeta| \leq 2 c d(\xi)
$$

for some $c>0$ since $\omega(t)=o(t)$.

If $|\xi-\zeta| \leq|\zeta|$ then $|\xi| \leq 2|\zeta|$ and, by property $(\alpha)$ of $\omega$ and (3),

$$
\omega(\xi) \leq L(\omega(\zeta)+1) \leq L^{\prime}(|\operatorname{Im} \zeta|+1) \leq L^{\prime}(|\zeta-\xi|+1) \leq L^{\prime \prime} d(\xi),
$$

for some $L^{\prime}, L^{\prime \prime}>0$, since (3) implies that $d(\xi) \rightarrow+\infty$ (see [25, Prop. 2.2.1]).

(4) $\Rightarrow(3)$ : If $|\operatorname{Im} \zeta| \leq|\operatorname{Re} \zeta|$, then property $(\alpha)$ of $\omega$ and (4) imply that

$$
\omega(\zeta) \leq L(\omega(\operatorname{Re} \zeta)+1) \leq L^{\prime}(d(\operatorname{Re} \zeta)+1) \leq L^{\prime}(|\operatorname{Im} \zeta|+1)
$$

for some $L^{\prime}>0$.

If $|\operatorname{Re} \zeta| \leq|\operatorname{Im} \zeta|$ then, by property $(\alpha)$ of $\omega$,

$$
\omega(\zeta) \leq L(\omega(|\operatorname{Im} \zeta|)+1) \leq L^{\prime}(|\operatorname{Im} \zeta|+1)
$$

for some $L^{\prime}>0$ since $\omega(t)=o(t)$.

$(4) \Leftrightarrow(5)$ : It is straightforward because of $(2.3)$.

Remark 2.10. From Theorems 2.7 and 2.9 we immediately get the well-known result that $(\omega)$ hypoellipticity implies $\{\omega\}$-hypoellipticity and they both imply hypoellipticity by Theorem 2.4. 


\section{Characterization of $\omega$-micro-hypoellipticity with respect to the it- erates of an operator}

Let $\omega$ be a non-quasianalytic weight function and $P(D)$ a linear partial differential operator with constant coefficients. We want to characterize the functions in the class $\mathcal{E}_{*}^{P}$, for $*=(\omega)$ or $\{\omega\}$, by means of the Fourier transform. To this aim we use a Paley-Wiener theorem for these spaces of functions, that we borrow from [15]. Since our spaces $\mathcal{E}_{*}^{P}(\Omega)$ are slightly different from the analogous ones defined in [15], our Paley-Wiener theorem is only sligthly different from the one of [15], and therefore we shall present the suitable statement here, omiting the proof (see [15, Lemma 3.1]).

Lemma 3.1. Let $\omega$ be a non-quasianalytic weight function, $P$ a polynomial of degree $m, K$ a compact convex subset of $\mathbb{R}^{n}$ and $f \in \mathcal{D}\left(\mathbb{R}^{n}\right)$ with supp $f \subset K$. Then the following statements are equivalent:

(1) there exists $\lambda>0$ such that

$$
\int_{\mathbb{R}^{n}}|\widehat{f}(\xi)|^{2} e^{\lambda \omega\left(|P(\xi)|^{1 / m}\right)} d \xi<+\infty ;
$$

(2) there exists $\lambda, C>0$ such that

$$
\left\|P^{j}(D) f\right\|_{2, \mathbb{R}^{n}} \leq C e^{\lambda \varphi^{*}\left(\frac{j m}{\lambda}\right)} \quad \forall j \in \mathbb{N}_{0} .
$$

To be more precise, if (1) holds for some $\lambda_{0}>0$, then (2) holds for $\lambda=\lambda_{0} / 2$ and for $C:=$ $(2 \pi)^{-n / 2}\left(\int_{\mathbb{R}^{n}}|\widehat{f}(\xi)|^{2} e^{\lambda_{0} \omega\left(|P(\xi)|^{1 / m}\right)} d \xi\right)^{1 / 2}$.

Conversely, if (2) holds for some $\lambda>0$, then

$$
|\widehat{f}(\zeta)| \leq m(K)^{1 / 2} C D_{\lambda, \omega} e^{H_{K}(\operatorname{Im} \zeta)-\frac{\lambda}{2} \omega\left(|P(\zeta)|^{1 / m}\right)} \quad \forall \zeta \in \mathbb{C}^{n},
$$

for some $D_{\lambda, \omega}>0$ depending on $\lambda$ and $\omega$, where $m(K)$ is the Lebesgue measure of $K$ and $H_{K}(\cdot)$ the supporting function of $K$; therefore (1) holds for any $\lambda^{\prime}<\lambda$.

We present also, similarly as in [15, Thms 3.3 and 3.4], the following Paley-Wiener type theorem for functions in

for $*=(\omega)$ or $\{\omega\}$ :

$$
\mathcal{D}_{*}^{P}\left(\mathbb{R}^{n}\right)=\left\{f \in \mathcal{E}_{*}^{P}\left(\mathbb{R}^{n}\right): f \in \mathcal{D}\left(\mathbb{R}^{n}\right)\right\},
$$

Theorem 3.2. Let $P(\xi)$ be a hypoelliptic polynomial of degree $m$ and $\omega$ a non-quasianalytic weight function. If $f \in \mathcal{D}_{(\omega)}^{P}\left(\mathbb{R}^{n}\right)$ (resp. $f \in \mathcal{D}_{\{\omega\}}^{P}\left(\mathbb{R}^{n}\right)$ ) then its Fourier-Laplace transform $F(\zeta)=\widehat{f}(\zeta)$ is an entire function satisfying the following two conditions:

(i) there exist $C, A>0$ such that

$$
|F(\zeta)| \leq C e^{A|\zeta|} \quad \forall \zeta \in \mathbb{C}^{n}
$$

(ii) for every $\lambda>0$ (resp. there exists $\lambda>0$ ):

$$
\int_{\mathbb{R}^{n}}|F(\xi)|^{2} e^{\lambda \omega\left(|P(\xi)|^{\frac{1}{m}}\right)} d \xi<+\infty .
$$

Conversely, if $F$ is an entire function satisfying $(i)$ and $(i i)$, then $F(\zeta)=\widehat{f}(\zeta)$ for some $f \in \mathcal{D}_{(\omega)}^{P}\left(\mathbb{R}^{n}\right)$ (resp. $f \in \mathcal{D}_{\{\omega\}}^{P}\left(\mathbb{R}^{n}\right)$ ).

Now, we have all the tools to prove the following theorems of characterization of $\mathcal{E}_{(\omega)}^{P}$ and $\mathcal{E}_{\{\omega\}}^{P}$ :

Theorem 3.3. Let $P(D)$ be a hypoelliptic linear partial differential operator with constant coefficients, $\Omega$ an open subset of $\mathbb{R}^{n}, u \in \mathcal{D}^{\prime}(\Omega)$ and $x_{0} \in \Omega$. Let $\omega$ be a non-quasianalytic weight function such that $\omega\left(t^{\gamma}\right)=o(\sigma(t))$, as $t \rightarrow+\infty$, where $\gamma$ is the constant defined in $(2.5)$ and $\sigma(t)=t^{1 / s}$ for some $s>1$.

The following conditions are equivalent:

(1) $u \in \mathcal{E}_{(\omega)}^{P}(U)$ for some neighborhood $U$ of $x_{0}$. 
(2) There exists $\left\{f_{N}\right\}_{N \in \mathbb{N}} \subset \mathcal{E}^{\prime}(\Omega)$ such that $f_{N}=P(D)^{N} u$ in a neighborhood of $x_{0}$ and:

$$
\begin{aligned}
& \forall k \in \mathbb{N}, M \in \mathbb{R} \exists C_{k, M}>0: \\
& \left|\widehat{f}_{N}(\xi)\right| \leq C_{k, M} e^{k \varphi^{*}(N m / k)}(1+|\xi|)^{M} \quad \forall N \in \mathbb{N}, \xi \in \mathbb{R}^{n} .
\end{aligned}
$$

(3) There exists $\psi \in \mathcal{D}_{\{\sigma\}}(\Omega)$, with $\psi \equiv 1$ in a neighborhood of $x_{0}$, such that:

$$
\begin{aligned}
& \forall k \in \mathbb{N} \exists C_{k}>0: \\
& |\widehat{\psi u}(\xi)| \leq C_{k} e^{-k \omega\left(|P(\xi)|^{1 / m}\right)} \quad \forall \xi \in \mathbb{R}^{n} .
\end{aligned}
$$

(4) There exists $\psi \in \mathcal{D}_{\{\sigma\}}(\Omega)$, with $\psi \equiv 1$ in a neighborhood of $x_{0}$, such that:

$$
\begin{aligned}
& \forall k \in \mathbb{N}, \ell>0 \exists C_{k, \ell}>0: \\
& |P(\xi)|^{N}|\widehat{\psi u}(\xi)| \leq C_{k, \ell} e^{k \varphi^{*}(N m / k)}(1+|\xi|)^{-\ell} \quad \forall N \in \mathbb{N}_{0}, \xi \in \mathbb{R}^{n} .
\end{aligned}
$$

Theorem 3.4. Let $P(D)$ be a hypoelliptic linear partial differential operator with constant coefficients, $\Omega$ an open subset of $\mathbb{R}^{n}, u \in \mathcal{D}^{\prime}(\Omega)$ and $x_{0} \in \Omega$. Let $\omega$ be a non-quasianalytic weight function such that $\omega\left(t^{\gamma}\right)=o(\sigma(t))$, as $t \rightarrow+\infty$, where $\gamma$ is the constant defined in (2.5) and $\sigma(t)=t^{1 / s}$ for some $s>1$.

Then the following conditions are equivalent:

$\{1\} u \in \mathcal{E}_{\{\omega\}}^{P}(U)$ for some neighborhood $U$ of $x_{0}$.

$\{2\}$ There exists $\left\{f_{N}\right\}_{N \in \mathbb{N}} \subset \mathcal{E}^{\prime}(\Omega)$ such that $f_{N}=P(D)^{N} u$ in a neighborhood of $x_{0}$ and:

$$
\begin{aligned}
& \exists k \in \mathbb{N}, \forall M \in \mathbb{R} \exists C_{M}>0: \\
& \left|\widehat{f}_{N}(\xi)\right| \leq C_{M} e^{\frac{1}{k} \varphi^{*}(N m k)}(1+|\xi|)^{M} \quad \forall N \in \mathbb{N}, \xi \in \mathbb{R}^{n} .
\end{aligned}
$$

$\{3\}$ There exists $\psi \in \mathcal{D}_{\{\sigma\}}(\Omega)$, with $\psi \equiv 1$ in a neighborhood of $x_{0}$, such that:

$$
\begin{aligned}
& \exists k \in \mathbb{N}, C>0: \\
& |\widehat{\psi u}(\xi)| \leq C e^{-\frac{1}{k} \omega\left(|P(\xi)|^{1 / m}\right)} \quad \forall \xi \in \mathbb{R}^{n} .
\end{aligned}
$$

$\{4\}$ There exists $\psi \in \mathcal{D}_{\{\sigma\}}(\Omega)$, with $\psi \equiv 1$ in a neighborhood of $x_{0}$, such that:

$$
\begin{aligned}
& \exists k \in \mathbb{N}, \forall \ell>0 \exists C_{\ell}>0: \\
& |P(\xi)|^{N}|\widehat{\psi u}(\xi)| \leq C_{\ell} e^{\frac{1}{k} \varphi^{*}(N m k)}(1+|\xi|)^{-\ell} \quad \forall N \in \mathbb{N}_{0}, \xi \in \mathbb{R}^{n} .
\end{aligned}
$$

Before proving Theorems 3.3 and 3.4 we need the following lemma:

Lemma 3.5. Let $\Gamma \subseteq \mathbb{R}^{n}$ be a cone, $P(D)$ a linear partial differential operator of order $m$ with constant coefficients, $\Omega$ an open subset of $\mathbb{R}^{n}, u \in \mathcal{D}^{\prime}(\Omega), \psi \in \mathcal{D}(\Omega)$. Then:

(1) Beurling case. The following two conditions are equivalent:

(1.a) for every $k \in \mathbb{N}$ there exists $C_{k}>0$ such that

$$
|\widehat{\psi u}(\xi)| \leq C_{k} e^{-k \omega\left(|P(\xi)|^{1 / m}\right)} \quad \forall \xi \in \Gamma ;
$$

(1.b) for every $k \in \mathbb{N}, \ell>0$ there exists $C_{k, \ell}>0$ such that

$$
|P(\xi)|^{N}|\widehat{\psi u}(\xi)| \leq C_{k, \ell} e^{k \varphi^{*}(N m / k)}(1+|\xi|)^{-\ell} \quad \forall N \in \mathbb{N}_{0}, \xi \in \Gamma .
$$

(2) Roumieu case. The following two conditions are equivalent:

(2.a) there exist $k \in \mathbb{N}$ and $C>0$ such that

$$
|\widehat{\psi u}(\xi)| \leq C e^{-\frac{1}{k} \omega\left(|P(\xi)|^{1 / m}\right)} \quad \forall \xi \in \Gamma ;
$$

(2.b) there exists $k \in \mathbb{N}$ such that for every $\ell>0$ there is $C_{\ell}>0$ s.t.

$$
|P(\xi)|^{N}|\widehat{\psi u}(\xi)| \leq C_{\ell} e^{\frac{1}{k} \varphi^{*}(N m k)}(1+|\xi|)^{-\ell} \quad \forall N \in \mathbb{N}_{0}, \xi \in \Gamma .
$$


Proof of Lemma 3.5. Beurling case. (1.a) $\Rightarrow(1 . b)$ : Since $P$ is hypoelliptic, by (2.4), (1.a), [3, Lemma 16(i)] and the convexity of $\varphi^{*}$, we have, for all $\ell>0$ and $N \in \mathbb{N}_{0}$,

$$
\begin{aligned}
|\xi|^{\ell}|P(\xi)|^{N}|\widehat{\psi u}(\xi)| & \leq \delta^{-\ell / d}|P(\xi)|^{\frac{\ell}{d}+N}|\widehat{\psi u}(\xi)| \\
& \left.\leq \delta^{-\ell / d}\left(|P(\xi)|^{\frac{1}{m}}\right)^{\frac{\ell m}{d}+m N} C_{2 k} e^{-2 k \omega\left(|P(\xi)|^{\frac{1}{m}}\right.}\right) \\
& \leq C_{k, \ell} e^{k \varphi^{*}\left(\frac{N m}{k}\right)} .
\end{aligned}
$$

$(1 . b) \Rightarrow(1 . a)$ : Assume first that $|P(\xi)|>1$. By (1.b) with $\ell=m$ we have that for every $k$ there exists a constant $C_{k, m}=C_{k}>0$ such that

$$
|\xi|^{m}|P(\xi)|^{N} e^{-k \varphi^{*}\left(\frac{N m}{k}\right)}|\widehat{\psi u}(\xi)| \leq C_{k} \quad \forall N \in \mathbb{N}, \xi \in \Gamma .
$$

Since $|P(\xi)| \leq c|\xi|^{m}$ for some $c>0$, we thus have that

$$
\sup _{N \in \mathbb{N}_{0}}\left\{\left(|P(\xi)|^{1 / m}\right)^{(N+1) m} e^{-k \varphi^{*}\left(\frac{N m}{k}\right)}\right\}|\widehat{\psi u}(\xi)| \leq C_{k}^{\prime}
$$

for some $C_{k}^{\prime}>0$. But for all $s>0$ there exists $N \in \mathbb{N}_{0}$ such that $N m \leq s<(N+1) m$, so that

$$
\begin{aligned}
\sup _{N \in \mathbb{N}_{0}}\left\{\left(|P(\xi)|^{1 / m}\right)^{(N+1) m} e^{-k \varphi^{*}\left(\frac{N m}{k}\right)}\right\} & \geq \sup _{s>0}\left\{\left(|P(\xi)|^{1 / m}\right)^{s} e^{-k \varphi^{*}\left(\frac{s}{k}\right)}\right\} \\
& =\exp \left\{k \varphi\left(\log |P(\xi)|^{1 / m}\right)\right\}=e^{k \omega\left(|P(\xi)|^{1 / m}\right)} .
\end{aligned}
$$

Substituting in (3.8) we have that

$$
e^{k \omega\left(|P(\xi)|^{1 / m}\right)}|\widehat{\psi u}(\xi)| \leq C_{k}^{\prime} \quad \forall \xi \in \Gamma \text { with }|P(\xi)|>1 .
$$

If $|P(\xi)| \leq 1$ then $\omega\left(|P(\xi)|^{1 / m}\right) \equiv 0$ and the thesis is trivial.

Roumieu case. It is similar to the Beurling case.

Proof of Theorem 3.3. (1) $\Leftrightarrow(2)$ was proved in [3, Prop. 6].

$(1) \Rightarrow(3)$ : Let $u \in \mathcal{E}_{(\omega)}^{P}(U)$ and take $\psi \in \mathcal{D}_{\{\sigma\}}(U)$ with $\psi \equiv 1$ in a neighborhood $V \subset U$ of $x_{0}$. Since $\omega\left(t^{\gamma}\right)=o(\sigma(t))$, by Proposition 17 of [3] we have that $\psi u \in \mathcal{D}_{(\omega)}^{P}(U)$.

By the Paley-Wiener Theorem 3.2, for all $\lambda>0$ there exists $C_{\lambda}>0$ such that

$$
\int_{\mathbb{R}^{n}}|\widehat{\psi u}(\xi)|^{2} e^{4 \lambda \omega\left(|P(\xi)|^{1 / m}\right)} d \xi \leq C_{\lambda}
$$

and hence, by Lemma 3.1 for $K=\operatorname{supp} \psi$,

$$
|\widehat{\psi u}(\zeta)| \leq m(K)^{1 / 2} C_{\lambda} D_{\lambda, \omega} e^{H_{K}(\operatorname{Im} \zeta)-\lambda \omega\left(|P(\zeta)|^{1 / m}\right)} \quad \forall \zeta \in \mathbb{C}^{n} .
$$

For $\lambda=k \in \mathbb{N}$ and $\zeta=\xi \in \mathbb{R}^{n}$ we thus obtain (3.3).

$(3) \Rightarrow(1)$ : From (3.3) it follows that for every $\lambda>0$

$$
C_{\lambda}:=\left(\int_{\mathbb{R}^{n}}|\widehat{\psi u}(\xi)|^{2} e^{2 \lambda \omega\left(|P(\xi)|^{1 / m}\right)} d \xi\right)^{1 / 2}<+\infty .
$$

Therefore, by Lemma 3.1, we obtain

$$
\left\|P^{j}(D)(\psi u)\right\|_{2, \mathbb{R}^{n}} \leq(2 \pi)^{-n / 2} C_{\lambda} e^{\lambda \varphi^{*}\left(\frac{j m}{\lambda}\right)} \quad \forall j \in \mathbb{N}_{0} .
$$

Since $\psi \equiv 1$ in a neighborhood $U$ of $x_{0}$, then for every compact $K \subset \subset U$

$$
\left\|P^{j}(D) u\right\|_{2, K}=\left\|P^{j}(D)(\psi u)\right\|_{2, K} \leq\left\|P^{j}(D)(\psi u)\right\|_{2, \mathbb{R}^{n}} \leq C_{\lambda}^{\prime} e^{\lambda \varphi^{*}\left(\frac{j m}{\lambda}\right)} \quad \forall j \in \mathbb{N}_{0},
$$

for some $C_{\lambda}^{\prime}>0$, i.e. $u \in \mathcal{E}_{(\omega)}^{P}(U)$.

$(3) \Leftrightarrow(4)$ follows from Lemma 3.5 for $\Gamma=\mathbb{R}^{n}$.

Proof of Theorem 3.4. It is analogous to that of Theorem 3.3 for $\lambda=1 / k$.

Let us now consider the wave front set with respect to the iterates of an operator. We recall the following definition from [3]: 
Definition 3.6. Let $P(D)$ be a hypoelliptic linear partial differential operator with constant coefficients, $\omega$ a non-quasinanlytic weight function, $\Omega$ an open subset of $\mathbb{R}^{n}, u \in \mathcal{D}^{\prime}(\Omega)$. We say that a point $\left(x_{0}, \xi_{0}\right) \in \Omega \times\left(\mathbb{R}^{n} \backslash\{0\}\right)$ is not in the $(\omega)$-wave front set $\mathrm{WF}_{(\omega)}^{P}(u)$ (resp. $\{\omega\}$-wave front set $\left.\mathrm{WF}_{\{\omega\}}^{P}(u)\right)$ with respect to the iterates of $P$, if there are a neighborhood $U$ of $x_{0}$, an open conic neighborhood $\Gamma$ of $\xi_{0}$ and a sequence $\left\{f_{N}\right\}_{N \in \mathbb{N}} \subset \mathcal{E}^{\prime}(\Omega)$ such that $(i)$ and (ii) (resp. (i) and (iii)) of the following conditions hold:

(i) $f_{N}=P(D)^{N} u$ in $U$.

(ii) Beurling case:

(a) there exist $M, C>0$ such that for all $k \in \mathbb{N}$ there is $C_{k}>0$ :

$$
\left|\widehat{f}_{N}(\xi)\right| \leq C_{k} C^{N}\left(e^{\frac{k}{N m} \varphi^{*}\left(\frac{N m}{k}\right)}+|\xi|\right)^{N m}(1+|\xi|)^{M} \quad \forall N \in \mathbb{N}, \xi \in \mathbb{R}^{n} ;
$$

(b) for every $\ell \in \mathbb{N}_{0}, k \in \mathbb{N}$ there exists $C_{k, \ell}>0$ such that

(iii) Roumieu case:

$$
\left|\widehat{f}_{N}(\xi)\right| \leq C_{k, \ell} e^{k \varphi^{*}(N m / k)}(1+|\xi|)^{-\ell} \quad \forall N \in \mathbb{N}, \xi \in \Gamma .
$$

(a) there exist $k \in \mathbb{N}, M, C>0$ such that

$$
\left|\widehat{f}_{N}(\xi)\right| \leq C^{N}\left(e^{\frac{1}{N m k} \varphi^{*}(N m k)}+|\xi|\right)^{N m}(1+|\xi|)^{M} \quad \forall N \in \mathbb{N}, \xi \in \mathbb{R}^{n} ;
$$

(b) there exists $k \in \mathbb{N}$ such that for every $\ell \in \mathbb{N}_{0}$ there is $C_{\ell}>0$ s.t.

$$
\left|\widehat{f}_{N}(\xi)\right| \leq C_{\ell} e^{\frac{1}{k} \varphi^{*}(N m k)}(1+|\xi|)^{-\ell} \quad \forall N \in \mathbb{N}, \xi \in \Gamma .
$$

Now we want to characterize the $\omega$-wave front set in terms of condition (3.3) for the Beurling case, and (3.6) for the Roumieu case. To do this, we first give the following definition of wave front set and we prove in Theorem 3.13 below its equivalence to the one of Definition 3.6:

Definition 3.7. Let $P(D)$ be a hypoelliptic linear partial differential operator with constant coefficients; let $\omega$ be a non-quasianalytic weight function with $\omega\left(t^{\gamma}\right)=o(\sigma(t))$, as $t \rightarrow+\infty$, where $\gamma$ is the constant defined in (2.5) and $\sigma(t)=t^{1 / s}$ for some $s>1$; let $\Omega$ be an open subset of $\mathbb{R}^{n}$ and $u \in \mathcal{D}^{\prime}(\Omega)$.

We say that $\left(x_{0}, \xi_{0}\right) \in \Omega \times\left(\mathbb{R}^{n} \backslash\{0\}\right)$ is not in the $(\omega)$-wave front set $\mathrm{WF}_{(\omega), P}(u)$ (resp. $\{\omega\}$-wave front set $\left.\mathrm{WF}_{\{\omega\}, P}(u)\right)$ with respect to the iterates of $P$, if there exist a neighborhood $U$ of $x_{0}$, an open conic neighborhood $\Gamma$ of $\xi_{0}$ and $\psi \in \mathcal{D}_{\{\sigma\}}(\Omega)$ with $\psi \equiv 1$ in $U$ such that the following condition $(i)$ (resp. (ii)) holds:

(i) Beurling case: For every $k \in \mathbb{N}$ there exists $C_{k}>0$ such that

$$
|\widehat{\psi u}(\xi)| \leq C_{k} e^{-k \omega\left(|P(\xi)|^{1 / m}\right)} \quad \forall \xi \in \Gamma .
$$

(ii) Roumieu case: There exist $k \in \mathbb{N}, C>0$ such that

$$
|\widehat{\psi u}(\xi)| \leq C e^{-\frac{1}{k} \omega\left(|P(\xi)|^{1 / m}\right)} \quad \forall \xi \in \Gamma .
$$

In order to prove that $\mathrm{WF}_{*}^{P}(u)=\mathrm{WF}_{*, P}(u)$, for $*=(\omega)$ or $\{\omega\}$, let's start by the following:

Lemma 3.8. Let $P(D)$ be a hypoelliptic linear partial differential operator of order $m$ with constant coefficients; let $\omega$ be a non-quasianalytic weight function such that $\omega\left(t^{\gamma}\right)=o(\sigma(t))$, as $t \rightarrow+\infty$, where $\gamma$ is the constant defined in (2.5) and $\sigma(t)=t^{1 / s}$ for some $s>1$; let $\Omega$ be an open subset of $\mathbb{R}^{n}$ and $u \in \mathcal{D}^{\prime}(\Omega)$. Then

$$
\mathrm{WF}_{*}^{P}(u) \subseteq \mathrm{WF}_{*, P}(u),
$$

for $*=(\omega)$ or $\{\omega\}$,

Proof. Beurling case. Let $\left(x_{0}, \xi_{0}\right) \notin \mathrm{WF}_{(\omega), P}(u)$. There exist then a neighborhood $U$ of $x_{0}$, an open conic neighborhood neighborhood $\Gamma$ of $\xi_{0}$ and $\psi \in \mathcal{D}_{\{\sigma\}}(\Omega)$ with $\psi \equiv 1$ in $U$ satisfying (3.13). Setting $f_{N}=P(D)^{N}(\psi u)$ we have that $f_{N} \in \mathcal{E}^{\prime}(\Omega), f_{N}=P(D)^{N} u$ in $U$ and

$$
\left|\widehat{f}_{N}(\xi)\right| \leq|P(\xi)|^{N}|\widehat{\psi u}(\xi)| \leq C^{N}(1+|\xi|)^{N m}(1+|\xi|)^{M} \quad \forall \xi \in \mathbb{R}^{n}
$$


for some $M>0$, since $\psi u \in \mathcal{E}^{\prime}\left(\mathbb{R}^{n}\right)$. Clearly (3.15) implies (3.9).

To prove (3.10) take $k \in \mathbb{N}, \ell>0$ and $\xi \in \Gamma$. By (2.4) and (3.13) we have

$$
\begin{aligned}
|\xi|^{\ell}\left|\widehat{f}_{N}(\xi)\right| & \leq \delta^{-\ell / d}|P(\xi)|^{\ell / d}|P(\xi)|^{N}|\widehat{\psi u}(\xi)| \\
& \leq \delta^{-\ell / d} C_{2 k}\left(|P(\xi)|^{1 / m}\right)^{\frac{\ell m}{d}+N m} e^{-2 k \omega\left(|P(\xi)|^{1 / m}\right)} \\
& \leq C_{k, \ell} e^{k \varphi^{*}\left(\frac{N m}{k}\right)},
\end{aligned}
$$

by $[3$, Lemma $16(\mathrm{i})]$ and the convexity of $\varphi^{*}$.

This proves (3.10) and hence $\left(x_{0}, \xi_{0}\right) \notin \mathrm{WF}_{(\omega)}^{P}(u)$.

Roumieu case. It's similar to the Beurling case.

We recall, from [9, Lemma 4] (see also [25, Proposition 3.4.4]), the following lemma that we shall need later:

Lemma 3.9. Let $\Gamma$ and $\Gamma^{\prime}$ be two cones in $\mathbb{R}^{n}$ such that $\Gamma^{\prime} \subset \subset \Gamma$ in the sense that $\Gamma^{\prime} \cap S^{n-1} \subset \subset$ $\Gamma \cap S^{n-1}$, where $S^{n-1}$ is the unit sphere in $\mathbb{R}^{n}$.

Then there exists a bounded $\phi \in \mathcal{E}_{(\omega)}\left(\mathbb{R}^{n}\right) \subset \mathcal{E}_{\{\omega\}}\left(\mathbb{R}^{n}\right)$ with $\operatorname{supp} \phi \subset \Gamma, \phi \equiv 1$ on $\Gamma^{\prime}$ (for large $|\xi|$ ), which is the symbol of a pseudo-differential operator $\phi(D)$ satisfying

$$
\widehat{\phi(D) u}(\xi)=\phi(\xi) \widehat{u}(\xi) \quad u \in \mathcal{D}_{(\omega)}\left(\mathbb{R}^{n}\right), \xi \in \mathbb{R}^{n} .
$$

Remark 3.10. Here, the definition of pseudodifferential operator is as in [9, Def. 3]. Then, we must consider the symbol of the operator $\phi(D)$ as $(2 \pi)^{-n} \phi(\xi)$ (compare with the beginning of the proof of [9, Theorem 2]).

We can now prove the following result:

Theorem 3.11. Let $P(D)$ be a hypoelliptic linear partial differential operator of order $m$ with constant coefficients; let $\omega$ be a non-quasianalytic weight function such that $\omega\left(t^{\gamma}\right)=o(\sigma(t))$, as $t \rightarrow+\infty$, where $\gamma$ is the constant defined in (2.5) and $\sigma(t)=t^{1 / s}$ for some $s>1$. Let $\Omega$ be an open subset of $\mathbb{R}^{n}$, $u \in \mathcal{E}^{\prime}(\Omega)$ and $\left(x_{0}, \xi_{0}\right) \in \Omega \times\left(\mathbb{R}^{n} \backslash\{0\}\right)$.

Then $\left(x_{0}, \xi_{0}\right) \notin \mathrm{WF}_{*}^{P}(u)$ if and only if there exists $\phi$ as in Lemma 3.9 such that $\left.\phi(D) u\right|_{V} \in \mathcal{E}_{*}^{P}(V)$ for some neighborhood $V$ of $x_{0}$, where $*=(\omega)$ or $\{\omega\}$.

Proof. Beurling case. Let $\left(x_{0}, \xi_{0}\right) \notin \mathrm{WF}_{(\omega)}^{P}(u)$. There exist a neighborhood $U$ of $x_{0}$, an open conic neighborhood $\Gamma$ of $\xi_{0}$ and a sequence $\left\{f_{N}\right\}_{N \in \mathbb{N}} \subset \mathcal{E}^{\prime}(\Omega)$ with $f_{N}=P(D)^{N} u$ in $U$ and satisfying (3.9) and (3.10).

Now, we consider a conic neigborhood $\Gamma^{\prime}$ of $\xi_{0}$ with $\Gamma^{\prime} \subset \subset \Gamma$. Take then $\phi$ as in Lemma 3.9 and define $h_{N}:=\phi(D) f_{N}$. Since $\phi(D)$ and $P(D)^{N}$ commute, we have that $h_{N}=P(D)^{N} \phi(D) u$ in a neighborhood of $x_{0}$ and $\widehat{h}_{N}(\xi)=\phi(\xi) \widehat{f}_{N}(\xi)$ satisfies (3.2) because of (3.10) and $\phi$ bounded with $\operatorname{supp} \phi \subset \Gamma$. Therefore $\left.\phi(D) u\right|_{V} \in \mathcal{E}_{(\omega)}^{P}(V)$ for a neighborhood $V$ of $x_{0}$ by Theorem 3.3.

Conversely, let us now assume $\left.\phi(D) u\right|_{V} \in \mathcal{E}_{(\omega)}^{P}(V)$, where $\phi$ is as in Lemma 3.9, for some neighborhood $V$ of $x_{0}$ and for some neighborhoods $\Gamma^{\prime} \subset \subset \Gamma$ of $\xi_{0}$. Take $\varphi \in \mathcal{D}_{\{\sigma\}}(V)$ with $\varphi \equiv 1$ in a neighborhood of $x_{0}$ and write

$$
\varphi u=\varphi \phi(D) u+\varphi(I-\phi(D)) u=: u_{1}+u_{2} .
$$

Since $\left.\phi(D) u\right|_{V} \in \mathcal{E}_{(\omega)}^{P}(V)$ by assumption and $\varphi \in \mathcal{D}_{\{\sigma\}}(V)$, we can apply [3, Prop. 17] to get $u_{1} \in \mathcal{E}_{(\omega)}^{P}(V)$. We thus have to prove that $\left(x_{0}, \xi_{0}\right) \notin \mathrm{WF}_{(\omega)}^{P}\left(u_{2}\right)$. By Lemma 3.8 it's enough to prove that $\left(x_{0}, \xi_{0}\right) \notin \mathrm{WF}_{(\omega), P}\left(u_{2}\right)$. To do this, we have to find $\psi \in \mathcal{D}_{\{\sigma\}}(\Omega)$ with $\psi \equiv 1$ in a neighborhood of $x_{0}$ and $\widehat{\psi u_{2}}(\xi)$ satisfying (3.13) in a conic neighborhood of $\xi_{0}$.

Taking $\psi \in \mathcal{D}_{\{\sigma\}}(\Omega)$ with $\psi \equiv 1$ on $\operatorname{supp} \varphi$ we have that $\psi u_{2}=u_{2}$ by the definition of $u_{2}$. Hence we will prove $(3.13)$ for $\widehat{u}_{2}$. We have

$$
\begin{aligned}
\widehat{u}_{2} & =\mathcal{F}(\varphi(I-\phi(D)) u)(\xi)=(2 \pi)^{-n}(\widehat{\varphi} *(1-\phi) \widehat{u})(\xi) \\
& =(2 \pi)^{-n} \int \widehat{\varphi}(\xi-\eta)(1-\phi)(\eta) \widehat{u}(\eta) d \eta .
\end{aligned}
$$


Let $\Lambda$ be a conic neigborhood of $\xi_{0}$ with $\bar{\Lambda} \subset \Gamma^{\prime}$, so that $(1-\phi)(\eta)=0$ in $\Lambda$. For fixed $\xi \in \Lambda$ we set

$$
\begin{aligned}
& A:=\left\{\eta \in \mathbb{R}^{n}:|\xi-\eta| \leq \delta(|\xi|+|\eta|)\right\} \\
& B:=\left\{\eta \in \mathbb{R}^{n}:|\xi-\eta|>\delta(|\xi|+|\eta|)\right\}
\end{aligned}
$$

for $\delta>0$ small enough so that $A \subset \Gamma^{\prime}$ and hence $(1-\phi)(\eta)=0$ in $A$.

Splitting the integral (3.17) we have, for $\xi \in \Lambda$ :

$$
\begin{aligned}
\widehat{u}_{2}(\xi) & =(2 \pi)^{-n} \int_{A} \widehat{\varphi}(\xi-\eta)(1-\phi)(\eta) \widehat{u}(\eta) d \eta+(2 \pi)^{-n} \int_{B} \widehat{\varphi}(\xi-\eta)(1-\phi)(\eta) \widehat{u}(\eta) d \eta \\
& =(2 \pi)^{-n} \int_{B} \widehat{\varphi}(\xi-\eta)(1-\phi)(\eta) \widehat{u}(\eta) d \eta .
\end{aligned}
$$

For $\xi \in \Lambda$ and $\eta \in B$, as $\varphi \in \mathcal{D}_{\{\sigma\}}(\Omega)$ and $\sigma(t)=t^{1 / s}$, there exist $C, C^{\prime}, \varepsilon, \varepsilon^{\prime}>0$ such that

$$
|\widehat{\varphi}(\xi-\eta)| \leq C e^{-\varepsilon \sigma(|\xi-\eta|)} \leq C^{\prime} e^{-\varepsilon^{\prime}(\sigma(|\xi|)+\sigma(|\eta|))} .
$$

Moreover, $|\widehat{u}(\eta)| \leq C(1+|\eta|)^{M}$ for some $C, M>0$ since $u \in \mathcal{E}^{\prime}(\Omega)$, and therefore

$$
\begin{aligned}
\left|\int_{B} \widehat{\varphi}(\xi-\eta)(1-\phi)(\eta) \widehat{u}(\eta) d \eta\right| & \leq C^{\prime} e^{-\varepsilon^{\prime}(\sigma(\xi))} \int|1-\phi(\eta)||\widehat{u}(\eta)| e^{-\varepsilon^{\prime}(\sigma(\eta))} d \eta \\
& \leq D e^{-\varepsilon^{\prime}(\sigma(\xi))} \int(1+|\eta|)^{M} e^{-\varepsilon^{\prime}(\sigma(\eta))} d \eta \\
& \leq D^{\prime} e^{-\varepsilon^{\prime}(\sigma(\xi))}
\end{aligned}
$$

for some $D, D^{\prime}>0$, because $1-\phi(\eta)$ is bounded.

Since $\omega\left(t^{\gamma}\right)=o(\sigma(t))$ with $\gamma \geq 1$ (see Remark 2.5), for every $k \in \mathbb{N}$ there exists $R_{k}>0$ such that for all $|\xi| \geq R_{k}$ :

$$
\omega\left(|P(\xi)|^{\frac{1}{m}}\right) \leq \omega(c|\xi|) \leq L(\omega(\xi)+1) \leq \frac{1}{k} \sigma(\xi)+L
$$

for some $c, L>0$, and hence, from (3.19) and (3.18), for every $k \in \mathbb{N}$ there is $D_{k}>0$ such that:

$$
\left|\widehat{u}_{2}(\xi)\right| \leq D_{k} e^{-k \omega\left(|P(\xi)|^{1 / m}\right)} \quad \forall \xi \in \Lambda,
$$

proving that $\left(x_{0}, \xi_{0}\right) \notin \mathrm{WF}_{(\omega), P}\left(u_{2}\right)$ and hence $\left(x_{0}, \xi_{0}\right) \notin \mathrm{WF}_{(\omega)}^{P}(u)$.

Roumieu case. The proof is analogous to that of the Beurling case. If $\left(x_{0}, \xi_{0}\right) \notin \mathrm{WF}_{\{\omega\}}^{P}(u)$ we find a neighborhood $U$ of $x_{0}$, an open conic neighborhood $\Gamma$ of $\xi_{0}$ and a sequence $\left\{f_{N}\right\}_{N \in \mathbb{N}} \subset \mathcal{E}^{\prime}(\Omega)$ satisfying (i), (iii)(a) and (iii)(b) of Definition 3.6. As in the Beurling case $h_{N}:=\phi(D) f_{N}$ satisfies the desired estimate (3.5), so that $\left.\phi(D) u\right|_{V} \in \mathcal{E}_{\{\omega\}}^{P}(V)$ for a neighborhood $V$ of $x_{0}$ by Theorem 3.4.

Conversely, if $\left.\phi(D) u\right|_{V} \in \mathcal{E}_{\{\omega\}}^{P}(V)$ we proceed as in the Beurling case and obtain that $u_{1}:=$ $\varphi \phi(D) u \in \mathcal{E}_{\{\omega\}}^{P}(V)$ by Proposition 17 of [3]. The proof that $\left(x_{0}, \xi_{0}\right) \notin \mathrm{WF}_{\{\omega\}}^{P}\left(u_{2}\right)$ is analogous to the one in the Beurling case, applying (3.20) for $k=1$.

Proposition 3.12. Let $P(D)$ be a hypoelliptic linear partial differential operator with constant coefficients; let $\omega$ be a non-quasianalytic weight function such that $\omega\left(t^{\gamma}\right)=o(\sigma(t))$, as $t \rightarrow+\infty$, where $\gamma$ is the constant defined in $(2.5)$ and $\sigma(t)=t^{1 / s}$ for some $s>1$; let $\Omega$ be an open subset of $\mathbb{R}^{n}$ and $u \in \mathcal{D}^{\prime}(\Omega)$. If $\psi \in \mathcal{D}_{\{\sigma\}}(\Omega)$, then

$$
\mathrm{WF}_{*}^{P}(\psi u) \subseteq \mathrm{WF}_{*}^{P}(u),
$$

for $*=(\omega)$ or $\{\omega\}$.

Proof. Beurling case. Let $\left(x_{0}, \xi_{0}\right) \notin \mathrm{WF}_{(\omega)}^{P}(u)$. First, we observe that we can assume $u \in \mathcal{E}^{\prime}(\Omega)$, since the definition of the wave front set is local. Then, there exist a neighborhood $V$ of $x_{0}$ and a conic neighborhood $\Gamma$ of $\xi_{0}$ such that

$$
(V \times \Gamma) \cap \mathrm{WF}_{(\omega)}^{P}(u)=\emptyset .
$$


For $\Gamma^{\prime} \subset \subset \Gamma$ there exists, by Theorem 3.11, a bounded $\phi \in \mathcal{E}_{(\omega)}\left(\mathbb{R}^{n}\right)$ with $\operatorname{supp} \phi \subset \Gamma, \phi \equiv 1$ in $\Gamma^{\prime}$ such that $\left.\phi(D) u\right|_{V^{\prime}} \in \mathcal{E}_{(\omega)}^{P}\left(V^{\prime}\right)$ for some neighborhood $V^{\prime} \subseteq V$ of $x_{0}$.

Let us then consider $\varphi \in \mathcal{D}_{\{\sigma\}}\left(V^{\prime}\right)$ with $\varphi \equiv 1$ in a neighborhood of $x_{0}$, and set

$$
\varphi \psi u=\varphi \psi \phi(D) u+\varphi \psi(I-\phi(D)) u=: u_{1}+u_{2} .
$$

Since $\varphi, \psi \in \mathcal{D}_{\{\sigma\}}(\Omega)$ we have, by [7, Prop. 4.4], that $\varphi \psi \in \mathcal{D}_{\{\sigma\}}(\Omega)$ and hence $u_{1} \in \mathcal{E}_{(\omega)}^{P}\left(V^{\prime}\right)$ by $[3$, Prop. 17] because $\left.\phi(D) u\right|_{V^{\prime}} \in \mathcal{E}_{(\omega)}^{P}\left(V^{\prime}\right)$.

Arguing as in the proof of Theorem 3.11 with $\varphi \psi \in \mathcal{D}_{\{\sigma\}}$ instead of $\varphi \in \mathcal{D}_{\{\sigma\}}$, we obtain that $\left(x_{0}, \xi_{0}\right) \notin \mathrm{WF}_{(\omega), P}\left(u_{2}\right)$ and hence $\left(x_{0}, \xi_{0}\right) \notin \mathrm{WF}_{(\omega)}^{P}\left(u_{2}\right)$ by Lemma 3.8.

Therefore $\left(x_{0}, \xi_{0}\right) \notin \mathrm{WF}_{(\omega)}^{P}(\psi u)$.

Roumieu case. It is similar to the Beurling case.

Theorem 3.13. Let $P(D)$ be a hypoelliptic linear partial differential operator of order $m$ with constant coefficients; let $\omega$ be a non-quasianalytic weight function such that $\omega\left(t^{\gamma}\right)=o(\sigma(t))$, as $t \rightarrow+\infty$, where $\gamma$ is the constant defined in $(2.5)$ and $\sigma(t)=t^{1 / s}$ for some $s>1$; let $\Omega$ be an open subset of $\mathbb{R}^{n}$ and $u \in \mathcal{D}^{\prime}(\Omega)$. Then

$$
\mathrm{WF}_{*}^{P}(u)=\mathrm{WF}_{*, P}(u),
$$

for $*=(\omega)$ or $\{\omega\}$,

Proof. The inclusion

$$
\mathrm{WF}_{*}^{P}(u) \subseteq \mathrm{WF}_{*, P}(u)
$$

has been proved in Lemma 3.8.

Let us prove the other inclusion.

Beurling case. Let $\left(x_{0}, \xi_{0}\right) \notin \mathrm{WF}_{(\omega)}^{P}(u)$. There exist a compact neighborhood $K$ of $x_{0}$ and a closed conic neighborhood $F$ of $\xi_{0}$ such that

$$
\mathrm{WF}_{(\omega)}^{P}(u) \cap(K \times F)=\emptyset .
$$

Take, according to [11, Lemma 2.2], $\chi_{N} \subset \mathcal{D}(K)$ with $\chi_{N} \equiv 1$ in a neighborhood $K^{\prime} \subset K$ of $x_{0}$, that satisfies

$$
\sup _{K}\left|D^{\alpha+\beta} \chi_{N}\right| \leq C_{\alpha}\left(C_{\alpha} N\right)^{|\beta|} \quad \forall \alpha, \beta \in \mathbb{N}_{0},|\beta| \leq N .
$$

Fix $\psi \in \mathcal{D}_{\{\sigma\}}\left(K^{\prime}\right)$. By Proposition 3.12

and, hence,

$$
\mathrm{WF}_{(\omega)}^{P}(\psi u) \subseteq \mathrm{WF}_{(\omega)}^{P}(u),
$$

$$
\mathrm{WF}_{(\omega)}^{P}(\psi u) \cap(K \times F)=\emptyset .
$$

Now, consider $g_{N}:=\chi_{N m p} P(D)^{N} \psi u$, for $p$ sufficiently large so that, by [3, Corollary 10], for every $k, \ell \in \mathbb{N}$ there exists $C_{k, \ell}>0$ such that

$$
\left|\widehat{g}_{N}(\xi)\right| \leq C_{k, \ell} e^{k \varphi^{*}\left(\frac{N m}{k}\right)}(1+|\xi|)^{-\ell} \quad \forall N \in \mathbb{N}, \xi \in F .
$$

Moreover,

$$
g_{N}=\chi_{N m p} P(D)^{N} \psi u=P(D)^{N} \psi u \quad \text { in } \mathbb{R}^{n},
$$

since $\chi_{N m p} \equiv 1$ on $\operatorname{supp} \psi$.

Therefore

$$
\left|P(\xi)^{N} \widehat{\psi u}(\xi)\right|=\left|\mathcal{F}\left(P(D)^{N} \psi u\right)(\xi)\right|=\left|\widehat{g}_{N}(\xi)\right| \leq C_{k, \ell} e^{k \varphi^{*}\left(\frac{N m}{k}\right)}(1+|\xi|)^{-\ell} \quad \forall N \in \mathbb{N}, \xi \in F .
$$

By Lemma 3.5, this implies that

$$
|\widehat{\psi u}(\xi)| \leq C_{k} e^{-k \omega\left(|P(\xi)|^{1 / m}\right)} \quad \forall \xi \in F,
$$

i.e. $\left(x_{0}, \xi_{0}\right) \notin \mathrm{WF}_{(\omega), P}(u)$. 
Roumieu case. It is similar to the Beurling case.

By Theorem 3.13 and Lemma 3.5 we can now prove the following characterizations of the wave front set with respect to the iterates:

Corollary 3.14. Let $P(D)$ be a hypoelliptic linear partial differential operator with constant coefficients; let $\omega$ be a non-quasianalytic weight function such that $\omega\left(t^{\gamma}\right)=o(\sigma(t))$, as $t \rightarrow+\infty$, where $\gamma$ is the constant defined in $(2.5)$ and $\sigma(t)=t^{1 / s}$ for some $s>1$; let $\Omega$ be an open subset of $\mathbb{R}^{n}, u \in \mathcal{D}^{\prime}(\Omega)$ and $\left(x_{0}, \xi_{0}\right) \in \Omega \times\left(\mathbb{R}^{n} \backslash\{0\}\right)$.

The following conditions are equivalent:

(1) $\left(x_{0}, \xi_{0}\right) \notin \mathrm{WF}_{(\omega)}^{P}(u)$.

(2) There exist a neighborhood $U$ of $x_{0}$, an open conic neighborhood $\Gamma$ of $\xi_{0}$ and $\psi \in \mathcal{D}_{\{\sigma\}}(\Omega)$ with $\psi \equiv 1$ in $U$ such that:

$$
\begin{aligned}
& \forall k \in \mathbb{N}, \exists C_{k}>0: \\
& |\widehat{\psi u}(\xi)| \leq C_{k} e^{-k \omega\left(|P(\xi)|^{1 / m}\right)} \quad \forall \xi \in \Gamma .
\end{aligned}
$$

(3) There exist a neighborhood $U$ of $x_{0}$, an open conic neighborhood $\Gamma$ of $\xi_{0}$ and $\psi \in \mathcal{D}_{\{\sigma\}}(\Omega)$ with $\psi \equiv 1$ in $U$ such that:

$$
\begin{aligned}
& \forall k, \ell \in \mathbb{N}, \exists C_{k, \ell}>0: \\
& |P(\xi)|^{N}|\widehat{\psi u}(\xi)| \leq C_{k, \ell} e^{k \varphi^{*}\left(\frac{N m}{k}\right)}(1+|\xi|)^{-\ell} \quad \forall N \in \mathbb{N}, \xi \in \Gamma .
\end{aligned}
$$

(4) There exist a neighborhood $U$ of $x_{0}$, an open conic neighborhood $\Gamma$ of $\xi_{0}$ and a bounded sequence $\left\{u_{N}\right\}_{N \in \mathbb{N}} \subset \mathcal{E}^{\prime}(\Omega)$ such that $u_{N}=u$ in $U$ and:

$$
\begin{aligned}
& \forall k \in \mathbb{N}, \ell \in \mathbb{N}_{0}, \exists C_{k, \ell}>0: \\
& |P(\xi)|^{N}\left|\widehat{u}_{N}(\xi)\right| \leq C_{k, \ell} e^{k \varphi^{*}\left(\frac{N m}{k}\right)}(1+|\xi|)^{-\ell} \quad \forall N \in \mathbb{N}, \xi \in \Gamma .
\end{aligned}
$$

Corollary 3.15. Let $P(D)$ be a hypoelliptic linear partial differential operator with constant coefficients; let $\omega$ be a non-quasianalytic weight function such that $\omega\left(t^{\gamma}\right)=o(\sigma(t))$, as $t \rightarrow+\infty$, where $\gamma$ is the constant defined in (2.5) and $\sigma(t)=t^{1 / s}$ for some $s>1$; let $\Omega$ be an open subset of $\mathbb{R}^{n}, u \in \mathcal{D}^{\prime}(\Omega)$ and $\left(x_{0}, \xi_{0}\right) \in \Omega \times\left(\mathbb{R}^{n} \backslash\{0\}\right)$.

The following conditions are equivalent:

(1) $\left(x_{0}, \xi_{0}\right) \notin \mathrm{WF}_{\{\omega\}}^{P}(u)$.

(2) There exist a neighborhood $U$ of $x_{0}$, an open conic neighborhood $\Gamma$ of $\xi_{0}$ and $\psi \in \mathcal{D}_{\{\sigma\}}(\Omega)$ with $\psi \equiv 1$ in $U$ such that:

$$
\begin{aligned}
& \exists k \in \mathbb{N}, C>0: \\
& |\widehat{\psi u}(\xi)| \leq C e^{-\frac{1}{k} \omega\left(|P(\xi)|^{1 / m}\right)} \quad \forall \xi \in \Gamma .
\end{aligned}
$$

(3) There exist a neighborhood $U$ of $x_{0}$, an open conic neighborhood $\Gamma$ of $\xi_{0}$ and $\psi \in \mathcal{D}_{\{\sigma\}}(\Omega)$ with $\psi \equiv 1$ in $U$ such that:

$$
\begin{aligned}
& \exists k \in \mathbb{N}, \forall \ell \in \mathbb{N}, \exists C_{\ell}>0: \\
& |P(\xi)|^{N}|\widehat{\psi u}(\xi)| \leq C_{\ell} e^{\frac{1}{k} \varphi^{*}(N m k)}(1+|\xi|)^{-\ell} \quad \forall N \in \mathbb{N}, \xi \in \Gamma .
\end{aligned}
$$

(4) There exist a neighborhood $U$ of $x_{0}$, an open conic neighborhood $\Gamma$ of $\xi_{0}$ and a bounded sequence $\left\{u_{N}\right\}_{N \in \mathbb{N}} \subset \mathcal{E}^{\prime}(\Omega)$ such that $u_{N}=u$ in $U$ and:

$\exists k \in \mathbb{N}, \forall \ell \in \mathbb{N}_{0}, \exists C_{\ell}>0$ :

$$
|P(\xi)|^{N}\left|\widehat{u}_{N}(\xi)\right| \leq C_{\ell} e^{\frac{1}{k} \varphi^{*}(N m k)}(1+|\xi|)^{-\ell} \quad \forall N \in \mathbb{N}, \xi \in \Gamma .
$$

Proof of Corollary 3.14. (1) $\Leftrightarrow$ (2) follows from Theorem 3.13.

(2) $\Leftrightarrow(3)$ follows from Lemma 3.5.

$(3) \Rightarrow(4)$ : Taking $u_{N}=\psi u$ we have that $\left\{u_{N}\right\}_{N \in \mathbb{N}}$ is a bounded sequence in $\mathcal{E}^{\prime}(\Omega), u_{N}=u$ in $U$ and (3.24) implies (3.25) by the choice of $u_{N}$. 
(4) $\Rightarrow(1)$ : Taking $f_{N}=P(D)^{N} u_{N}$ we have that $f_{N}=P(D)^{N} u$ in $U$ and, by (3.25),

$$
\left|\widehat{f}_{N}(\xi)\right|=|P(\xi)|^{N}\left|\widehat{u}_{N}(\xi)\right| \leq C_{k, \ell} e^{k \varphi^{*}\left(\frac{N m}{k}\right)}(1+|\xi|)^{-\ell} \quad \forall \xi \in \Gamma,
$$

giving condition (3.10).

Finally, since $\left\{u_{N}\right\}_{N \in \mathbb{N}}$ is a bounded sequence in $\mathcal{E}^{\prime}(\Omega)$, there exist $c, M>0$ such that

$$
\left|\widehat{u}_{N}(\xi)\right| \leq c(1+|\xi|)^{M} \quad \forall \xi \in \mathbb{R}^{n}
$$

and hence

$$
\begin{aligned}
\left|\widehat{f}_{N}(\xi)\right| & \leq|P(\xi)|^{N}\left|\widehat{u}_{N}(\xi)\right| \leq C^{N}(1+|\xi|)^{m N} c(1+|\xi|)^{M} \\
& \leq \tilde{C}^{N}\left(e^{\frac{k}{N m} \varphi^{*}\left(\frac{N m}{k}\right)}+|\xi|\right)^{N m}(1+|\xi|)^{M} \quad \forall \xi \in \mathbb{R}^{n},
\end{aligned}
$$

for some $C, \tilde{C}>0$, proving also (3.9).

Therefore $\left(x_{0}, \xi_{0}\right) \notin \mathrm{WF}_{(\omega)}^{P}(u)$.

Proof of Corollary 3.15. Its similar to the Beurling case, Corollary 3.14.

The new characterization of $\mathrm{WF}_{*}^{P}(u)$ given by Corollaries 3.14 and 3.15 allows to complete Theorem 18 of [3], obtaining the existence of a distribution with prescribed $\omega$-wave front set with respect to the iterates:

Theorem 3.16. Let $P(D)$ be a linear partial differential operator of order $m$ with constant coefficients which is hypoelliptic, but not elliptic. Let $\omega$ be a non-quasi-analytic weight function such that $\omega\left(t^{b}\right)=$ $o(\bar{\sigma}(t))$, as $t \rightarrow+\infty$, where $\bar{\sigma}(t)=t^{1 / s}$ for some $s>1$ and $b=\max \{\gamma, 3 / 2\}$, with $\gamma$ defined in $(2.5)$.

Given an open subset $\Omega$ of $\mathbb{R}^{n}$ and a closed conic subset $S$ of $\Omega \times\left(\mathbb{R}^{n} \backslash\{0\}\right)$, there exists $u \in \mathcal{D}^{\prime}(\Omega)$ with

$$
\mathrm{WF}_{*}^{P}(u)=S,
$$

for $*=(\omega)$ or $\{\omega\}$

Proof. We assume, without loss of generality, that $\Omega=\mathbb{R}^{n}$; we construct the same distribution $u \in$ $\mathcal{D}^{\prime}\left(\mathbb{R}^{n}\right)$ constructed in [3, Thm. 18] and follow the ideas therein and in [12, Thm. 8.1.4].

We choose a sequence $\left(x_{k}, \theta_{k}\right) \in S$ with $\left|\theta_{k}\right|=1$ so that every $(x, \theta) \in S$ with $|\theta|=1$ is the limit of a subsequence.

We set $\sigma(t):=\omega\left(t^{3 / 2}\right)$ and separate the Beurling and the Roumieu cases.

Beurling case: Take $\phi \in \mathcal{D}_{(\omega)}\left(\mathbb{R}^{n}\right)$ with $\widehat{\phi}(0)=1$ and define, as in (158) of [3],

$$
u(x)=\sum_{k=1}^{+\infty} e^{-\sigma\left(k^{d / m}\right)} \phi\left(k\left(x-x_{k}\right)\right) e^{i k^{3}\left\langle x, \theta_{k}\right\rangle},
$$

where $d$ is the constant of $(2.4)$ with $0<d<m$ since $P$ is not elliptic by assumption. This is a continuous function in $\mathbb{R}^{n}$ and it was already proved in $[3, \mathrm{Thm} .18]$ that

$$
\emptyset \neq \mathrm{WF}_{(\omega)}^{P}(u) \subseteq S .
$$

Let us now prove the other inclusion. Fix $\left(x_{0}, \xi_{0}\right) \in S$ and assume by contradiction that $\left(x_{0}, \xi_{0}\right) \notin$ $\mathrm{WF}_{(\omega)}^{P}(u)$. Then, by Corollary 3.14, there exist a neighborhood $U$ of $x_{0}$, an open conic neighborhood $\Gamma$ of $\xi_{0}$ and $\psi \in \mathcal{D}_{\{\bar{\sigma}\}}\left(\mathbb{R}^{n}\right)$ with $\psi \equiv 1$ in $U$ such that (3.24) is satisfied.

Set then

$$
\phi_{k}(y)=\psi\left(\frac{y}{k}+x_{k}\right) \phi(y) .
$$

Since $\sigma(t)=o(\bar{\sigma}(t))$ by assumption, we have that $\mathcal{D}_{\{\bar{\sigma}\}}\left(\mathbb{R}^{n}\right) \subseteq \mathcal{D}_{(\sigma)}\left(\mathbb{R}^{n}\right)$ by [7, Prop. 4.7] and therefore $\left\{\phi_{k}\right\}_{k \in \mathbb{N}}$ is a bounded sequence in $\mathcal{D}_{(\sigma)}\left(\mathbb{R}^{n}\right)$, taking into account that supp $\phi_{k} \subseteq \operatorname{supp} \phi$ for all $k \in \mathbb{N}$. By [7, Prop. 3.4], for each $h \in \mathbb{N}$ there is $C_{h}>0$ such that

$$
\left|\widehat{\phi}_{j}(\xi)\right| \leq C_{h} e^{-h \sigma(\xi)} \quad \forall j \in \mathbb{N}, \xi \in \mathbb{R}^{n} .
$$


Moreover, following [3], we have

$$
\begin{aligned}
\mathcal{F}\left(P(D)^{N}(\psi u)\right)(\xi) & =\sum_{j=1}^{+\infty} e^{-\sigma\left(j^{d / m}\right)} P(\xi)^{N} \mathcal{F}\left(\phi_{j}\left(j\left(x-x_{j}\right)\right) e^{i j^{3}\left\langle x, \theta_{j}\right\rangle}\right) \\
& =\sum_{j=1}^{+\infty} e^{-\sigma\left(j^{d / m}\right)} P(\xi)^{N} j^{-n} \widehat{\phi}_{j}\left(\frac{\xi-j^{3} \theta_{j}}{j}\right) e^{i\left\langle x_{j}, j^{3} \theta_{j}-\xi\right\rangle}
\end{aligned}
$$

If $x_{k}$ is close to $x_{0}$ and $k$ is large enough, then $\phi_{k}=\phi$ and

$$
\begin{aligned}
\left|\mathcal{F}\left(P(D)^{N}(\psi u)\right)\left(k^{3} \theta_{k}\right)\right|= & \mid e^{-\sigma\left(k^{d / m}\right)} k^{-n} P\left(k^{3} \theta_{k}\right)^{N} \\
& +\sum_{j \neq k} e^{-\sigma\left(j^{d / m}\right)} j^{-n} P\left(k^{3} \theta_{k}\right)^{N} \widehat{\phi}_{j}\left(\frac{k^{3} \theta_{k}-j^{3} \theta_{j}}{j}\right) e^{i\left\langle x_{j}, j^{3} \theta_{j}-k^{3} \theta_{k}\right\rangle} \mid \\
\geq & \left|P\left(k^{3} \theta_{k}\right)\right|^{N}\left(e^{-\sigma\left(k^{d / m}\right)} k^{-n}-\sum_{j \neq k} e^{-\sigma\left(j^{d / m}\right)} j^{-n} C_{h} e^{-h \sigma\left(\frac{k^{3} \theta_{k}-j^{3} \theta_{j}}{j}\right)}\right) \\
\geq & \delta^{N} k^{3 N d}\left(e^{-\sigma\left(k^{d / m}\right)} k^{-n}-C_{h}^{\prime} e^{-h \sigma(k)}\right),
\end{aligned}
$$

for some $C_{h}^{\prime}>0$, because of $(3.30),(2.4)$ and

$$
\left|k^{3} \theta_{k}-j^{3} \theta_{j}\right| \geq\left|k^{3}-j^{3}\right| \geq k^{2}+k j+j^{2} \geq k j \quad \text { if } k \neq j .
$$

But for every fixed $h \geq 2$ there exists $k_{0} \in \mathbb{N}$ such that

$$
\frac{\log C_{h}^{\prime}}{\sigma(k)}+\frac{\log 2}{\sigma(k)}+\frac{n \log k}{\sigma(k)}+\frac{\sigma\left(k^{d / m}\right)}{\sigma(k)} \leq h \quad \forall k \geq k_{0}
$$

since $\log k=o(\sigma(k))$ and $0<d<m$.

Therefore

$$
\left|\mathcal{F}\left(P(D)^{N}(\psi u)\right)\left(k^{3} \theta_{k}\right)\right| \geq \frac{1}{2} \delta^{N} k^{3 N d} k^{-n} e^{-\sigma\left(k^{d / m}\right)} \quad \forall k \geq k_{0} .
$$

On the other hand, by (3.24):

$$
\left|\mathcal{F}\left(P(D)^{N}(\psi u)\right)\left(k^{3} \theta_{k}\right)\right|=\left|P\left(k^{3} \theta_{k}\right)\right|^{N}\left|\widehat{\psi u}\left(k^{3} \theta_{k}\right)\right| \leq C_{h, \ell} e^{h \varphi^{*}\left(\frac{N m}{h}\right)}\left(1+\left|k^{3} \theta_{k}\right|\right)^{-\ell} .
$$

But (3.31) and (3.32) give a contradiction for $k$ large enough (see [3] for more details). Therefore $\left(x_{0}, \xi_{0}\right) \in \mathrm{WF}_{(\omega)}^{P}(u)$ and $S \subseteq \mathrm{WF}_{(\omega)}^{P}(u)$.

Roumieu case: Take $\phi \in \mathcal{D}_{\{\sigma\}}\left(\mathbb{R}^{n}\right)$ with $\widehat{\phi}(0)=1$; choose, by Lemma 1.7 of [7], a non-quasianalytic weight function $\alpha(t)$ such that $\log t=o(\alpha(t))$ and $\alpha(t)=o(\sigma(t))$ for $t \rightarrow+\infty$, and define, as in (138) of [3],

$$
u(x)=\sum_{k=1}^{+\infty} e^{-\frac{\sigma(k d / m)}{\alpha\left(k^{d / m}\right)} \log k} \phi\left(k\left(x-x_{k}\right)\right) e^{i k^{3}\left\langle x, \theta_{k}\right\rangle} .
$$

This is a continuous function in $\mathbb{R}^{n}$ and it was already proved in [3, Thm. 18] that

$$
\emptyset \neq \mathrm{WF}_{\{\omega\}}^{P}(u) \subseteq S .
$$

Let us now prove the other inclusion. Fix $\left(x_{0}, \xi_{0}\right) \in S$ and assume by contradiction that $\left(x_{0}, \xi_{0}\right) \notin$ $\mathrm{WF}_{\{\omega\}}^{P}(u)$. Then, by Corollary 3.15, there exist a neighborhood $U$ of $x_{0}$, an open conic neighborhood $\Gamma$ of $\xi_{0}$ and $\psi \in \mathcal{D}_{\{\bar{\sigma}\}}\left(\mathbb{R}^{n}\right)$ with $\psi \equiv 1$ in $U$ such that (3.27) is satisfied.

Define, as in the Beurling case, $\phi_{k}(y)=\psi\left(\frac{y}{k}+x_{k}\right) \phi(y) \in \mathcal{D}_{\{\sigma\}}\left(\mathbb{R}^{n}\right)$ such that, by [7, Prop. 3.4], there exists $C, h>0$ :

$$
\left|\widehat{\phi}_{j}(\xi)\right| \leq C e^{-\frac{1}{h} \sigma(\xi)} \quad \forall j \in \mathbb{N}, \xi \in \mathbb{R}^{n} .
$$


Then, if $x_{k}$ is close to $x_{0}$ and $k$ is large enough,

$$
\begin{aligned}
\left|\mathcal{F}\left(P(D)^{N}(\psi u)\right)\left(k^{3} \theta_{k}\right)\right|= & \mid e^{-\frac{\sigma\left(k^{d / m}\right)}{\alpha\left(k^{d / m}\right)} \log k} k^{-n} P\left(k^{3} \theta_{k}\right)^{N} \\
& +\sum_{j \neq k} e^{-\frac{\sigma\left(j^{d / m}\right)}{\left.\alpha j^{d / m}\right)} \log j} j^{-n} P\left(k^{3} \theta_{k}\right)^{N} \widehat{\phi}_{j}\left(\frac{k^{3} \theta_{k}-j^{3} \theta_{j}}{j}\right) e^{i\left\langle x_{j}, j^{3} \theta_{j}-k^{3} \theta_{k}\right\rangle} \mid \\
\geq & \left|P\left(k^{3} \theta_{k}\right)^{N}\right|\left(e^{-\frac{\sigma\left(k^{d / m}\right)}{\alpha\left(k^{d / m}\right)} \log k} k^{-n}\right. \\
& \left.-\sum_{j \neq k} e^{-\frac{\sigma\left(j^{d / m}\right)}{\alpha\left(j^{d / m}\right)} \log j} j^{-n} C e^{-\frac{1}{h} \sigma\left(\frac{k^{3} \theta_{k}-j^{3} \theta_{j}}{j}\right)}\right) \\
\geq & \delta^{N} k^{3 N d}\left(e^{-\frac{\sigma\left(k^{d / m}\right)}{\alpha\left(k^{d / m}\right)} \log k} k^{-n}-C^{\prime} e^{-\frac{1}{h} \sigma(k)}\right) \\
\geq & \frac{1}{2} \delta^{N} k^{3 N d} e^{-\frac{\sigma\left(k^{d / m}\right)}{\alpha\left(k^{d / m}\right)} \log k} k^{-n}
\end{aligned}
$$

$$
\frac{\log \left(2 C^{\prime}\right)}{\sigma(k)}+\frac{\sigma\left(k^{d / m}\right)}{\sigma(k)} \frac{\log k}{\alpha\left(k^{d / m}\right)}+\frac{n \log k}{\sigma(k)} \leq \frac{1}{h}
$$

if $k$ is sufficiently large, since $\log k=o(\alpha(k)), \log k=o(\sigma(k))$ and $0<d<m$.

On the other hand, by (3.27) there exists $h \in \mathbb{N}$ such that

$$
\left|\mathcal{F}\left(P(D)^{N}(\psi u)\right)\left(k^{3} \theta_{k}\right)\right|=\left|P\left(k^{3} \theta_{k}\right)\right|^{N}\left|\widehat{\psi u}\left(k^{3} \theta_{k}\right)\right| \leq C_{\ell} e^{\frac{1}{h} \varphi^{*}(N m h)}\left(1+\left|k^{3} \theta_{k}\right|\right)^{-\ell}
$$

which contradicts (3.33) (see [3] for more details).

Therefore $\left(x_{0}, \xi_{0}\right) \in \mathrm{WF}_{\{\omega\}}^{P}(u)$ and $\mathrm{WF}_{\{\omega\}}^{P}(u)=S$.

Example 3.17. Let $\Omega$ be an open subset of $\mathbb{R}^{n}$ and $P(D)$ a linear partial differential operator with constant coefficients and of order $m$. By Theorems 4.1 and 4.8 of [1], for $*=(\omega)$ or $\{\omega\}$, we have

$$
\mathrm{WF}_{*}(u) \subseteq \mathrm{WF}_{*}(P u) \cup \Sigma, \quad u \in \mathcal{D}^{\prime}(\Omega),
$$

where

$$
\Sigma:=\left\{(x, \xi) \in \Omega \times \mathbb{R}^{n} \backslash\{0\}: P_{m}(\xi)=0\right\}
$$

is the characteristic set of $P$ (here $P_{m}$ is the principal part of $P$ ).

This implies that if $u$ is a solution of $P(D) u=f$, then

$$
\mathrm{WF}_{*}(u) \subseteq \Sigma, \quad \text { for each } f \in \mathcal{E}_{*}(\Omega),
$$

since $\mathrm{WF}_{*}(f)=\emptyset$ for $f \in \mathcal{E}_{*}(\Omega)$.

Now, by Theorem 13 of [3]

$$
\mathrm{WF}_{*}(u) \subseteq \mathrm{WF}_{*}^{P}(u) \cup \Sigma, \quad u \in \mathcal{D}^{\prime}(\Omega),
$$

so that we can improve (3.35) saying that

$$
\mathrm{WF}_{*}(u) \subseteq \Sigma \quad \text { if } f \in \mathcal{E}_{*}^{P}(\Omega) .
$$

Indeed, if $f \in \mathcal{E}_{*}^{P}(\Omega) \backslash \mathcal{E}_{*}(\Omega)$ then $\mathrm{WF}_{*}^{P}(f)=\emptyset$ and hence

$$
\mathrm{WF}_{*}(u) \subseteq \mathrm{WF}_{*}^{P}(u) \cup \Sigma=\mathrm{WF}_{*}^{P}(P u) \cup \Sigma=\mathrm{WF}_{*}^{P}(f) \cup \Sigma=\Sigma .
$$

Let us now prove a result that establishes the relationship between the wave front set in the Beurling class and in the Roumieu class. See also [1, Proposition 4.5] and [9, Proposition 2] for similar results in this setting for the usual wave front set, even for quasianalytic weight functions. 
Proposition 3.18. Let $P(D)$ be a hypoelliptic linear partial differential operator of order $m$ with constant coefficients, $\Omega$ an open subset of $\mathbb{R}^{n}$ and $u \in \mathcal{D}^{\prime}(\Omega)$. Let $\sigma_{0}$ and $\omega$ be two non-quasianalytic weight functions such that, for $t \rightarrow+\infty, \sigma_{0}(t)=o(\omega(t))$ and $\omega\left(t^{\gamma}\right)=o\left(t^{1 / s}\right)$ for $\gamma$ as in (2.5) and $s>1$. Then

$$
\mathrm{WF}_{\{\omega\}}^{P}(u)=\overline{\bigcup_{\sigma \in S} \mathrm{WF}_{(\sigma)}^{P}(u)},
$$

where $S:=\left\{\sigma\right.$ non-quasianalytic weight function: $\left.\sigma_{0} \leq \sigma=o(\omega)\right\}$.

Proof. Let us first prove that

$$
\overline{\bigcup_{\sigma \in S} \mathrm{WF}_{(\sigma)}^{P}(u)} \subseteq \mathrm{WF}_{\{\omega\}}^{P}(u)
$$

To do this, we fix $\left(x_{0}, \xi_{0}\right) \notin \mathrm{WF}_{\{\omega\}}^{P}(u)$. By Corollary 3.15 there exist a neighborhood $U$ of $x_{0}$, an open conic neighborhood $\Gamma$ of $\xi_{0}$ and $\psi \in \mathcal{D}_{\left\{t^{1 / s}\right\}}\left(\mathbb{R}^{n}\right)$ with $\psi \equiv 1$ in $U$ such that (3.26) is satisfied for some $k_{0} \in \mathbb{N}$ and $C>0$.

If $\sigma(t)=o(\omega(t))$, then for every $k \in \mathbb{N}$ there exists $t_{k}>0$ such that

$$
k \sigma(t) \leq \frac{1}{k_{0}} \omega(t) \quad \forall t \geq t_{k} .
$$

Since $P$ is hypoelliptic there exists then $R_{k}>0$ such that

$$
k \sigma\left(|P(\xi)|^{\frac{1}{m}}\right) \leq \frac{1}{k_{0}} \omega\left(|P(\xi)|^{\frac{1}{m}}\right) \quad \forall|\xi| \geq R_{k},
$$

and therefore there exists $C_{k}>0$ such that, by (3.26),

$$
|\widehat{\psi u}(\xi)| \leq C e^{-\frac{1}{k_{0}} \omega\left(|P(\xi)|^{\frac{1}{m}}\right)} \leq C_{k} e^{-k \sigma\left(|P(\xi)|^{\frac{1}{m}}\right)} \quad \forall \xi \in \mathbb{R}^{n} .
$$

This proves, by Corollary 3.14 , that $\left(x_{0}, \xi_{0}\right) \notin \mathrm{WF}_{(\sigma)}^{P}(u)$ and hence

$$
\bigcup_{\sigma \in S} \mathrm{WF}_{(\sigma)}^{P}(u) \subseteq \mathrm{WF}_{\{\omega\}}^{P}(u) .
$$

Since the wave front set $\mathrm{WF}_{\{\omega\}}^{P}(u)$ is always a closed set, we have the inclusion (3.36).

Let us prove the other inclusion. Take $\left(x_{0}, \xi_{0}\right) \notin \overline{\bigcup_{\sigma \in S} \mathrm{WF}_{(\sigma)}^{P}(u)}$. Then there exist a compact neighborhood $K$ of $x_{0}$ and a closed conic neighborhood $F$ of $\xi_{0}$ such that

$$
(K \times F) \cap \overline{\bigcup_{\sigma \in S} \mathrm{WF}_{(\sigma)}^{P}(u)}=\emptyset .
$$

Take $\chi_{N} \in \mathcal{D}(K)$ with $\chi_{N} \equiv 1$ in a neighborhood $K^{\prime} \subset K$ of $x_{0}$ which satisfies (3.21). Take then $\psi \in \mathcal{D}_{\left\{t^{1 / s}\right\}}\left(K^{\prime}\right)$. By Proposition 3.12

$$
\mathrm{WF}_{(\sigma)}^{P}(\psi u) \subseteq \mathrm{WF}_{(\sigma)}^{P}(u) \quad \forall \sigma \in S
$$

and hence

$$
(K \times F) \cap \mathrm{WF}_{(\sigma)}^{P}(\psi u)=\emptyset \quad \forall \sigma \in S .
$$

Consider then $g_{N}:=\chi_{N m p} P(D)^{N} \psi u$, for $p$ sufficiently large so that, by [3, Cor. 10]:

$$
\begin{aligned}
& \forall \sigma \in S, \forall k, \ell \in \mathbb{N} \exists C_{k, \ell, \sigma}>0 \text { s.t. } \\
& \left|\widehat{g}_{N}(\xi)\right| \leq C_{k, \ell, \sigma} e^{k \varphi_{\sigma}^{*}\left(\frac{N m}{k}\right)}(1+|\xi|)^{-\ell} \quad \forall \xi \in F,
\end{aligned}
$$

where $\varphi_{\sigma}^{*}$ is the Young conjugate of $\varphi_{\sigma}(t)=\sigma\left(e^{t}\right)$.

But

$$
g_{N}=\chi_{N m p} P(D)^{N} \psi u=P(D)^{N} \psi u \quad \text { in } \mathbb{R}^{n}
$$


since $\chi_{N m p} \equiv 1$ on $\operatorname{supp} \psi$. Therefore

$$
\left|P(\xi)^{N} \widehat{\psi u}(\xi)\right|=\left|\widehat{g}_{N}(\xi)\right| \leq C_{k, \ell, \sigma} e^{k \varphi_{\sigma}^{*}\left(\frac{N m}{k}\right)}(1+|\xi|)^{-\ell} \quad \forall \xi \in F,
$$

which implies, by Lemma 3.5:

$$
|\widehat{\psi u}(\xi)| \leq C_{k, \sigma} e^{-k \sigma\left(|P(\xi)|^{1 / m}\right)} \quad \forall \xi \in F .
$$

We want to prove that there exists $\bar{k} \in \mathbb{N}$ such that (3.26) is satisfied on $F$ (this would imply, by Corollary 3.15 , that $\left.\left(x_{0}, \xi_{0}\right) \notin \mathrm{WF}_{\{\omega\}}^{P}(u)\right)$. We argue by contradiction and assume that for every $n \in \mathbb{N}$ there exists $\xi_{n} \in F$ such that

$$
\left|P\left(\xi_{n}\right)\right| \geq \delta\left|\xi_{n}\right|^{d} \rightarrow+\infty
$$

and

$$
\left|\widehat{\psi u}\left(\xi_{n}\right)\right| \geq n e^{-\frac{1}{n} \omega\left(\left|P\left(\xi_{n}\right)\right|^{1 / m}\right)} \quad \forall n \in \mathbb{N} .
$$

Since $\sigma(t)=o(\omega(t))$, for $\sigma \in S$, for every $n \in \mathbb{N}$ there exists $k_{n} \geq n$ such that

$$
\frac{\sigma\left(\left|P\left(\xi_{k}\right)\right|^{1 / m}\right)}{\omega\left(\left|P\left(\xi_{k}\right)\right|^{1 / m}\right)}<\frac{1}{n} \quad \forall k \geq k_{n} .
$$

This would imply, together with (3.38), that

$$
\left|\widehat{\psi u}\left(\xi_{k_{n}}\right)\right| \geq k_{n} e^{-\frac{1}{k_{n}} \omega\left(\left|P\left(\xi_{k_{n}}\right)\right|^{1 / m}\right)} \geq n e^{-\frac{1}{n} \omega\left(\left|P\left(\xi_{k_{n}}\right)\right|^{1 / m}\right)}>n e^{-\sigma\left(\left|P\left(\xi_{k_{n}}\right)\right|^{1 / m}\right)},
$$

contradicting (3.37) for $k=1$.

Therefore $\left(x_{0}, \xi_{0}\right) \notin \mathrm{WF}_{\{\omega\}}^{P}(u)$ and the proposition is proved.

\section{Operators with constant strength}

We give some applications to linear partial differential operators with variable coefficients with constant strength. We recall, from [13], the following:

Definition 4.1. Let $P(D)$ and $Q(D)$ be two linear partial differential operators with constant coefficients. We say that $P$ is weaker than $Q$, and write $P \prec Q$, if there exists a constant $C>0$ such that

$$
\tilde{P}(\xi) \leq C \tilde{Q}(\xi) \quad \forall \xi \in \mathbb{R}^{n},
$$

where $\tilde{P}(\xi):=\sqrt{\sum_{\alpha}\left|D^{\alpha} P(\xi)\right|^{2}}$. We say that $P$ and $Q$ are equally strong if there exists a constant $C>0$ such that

$$
C^{-1} \tilde{P}(\xi) \leq \tilde{Q}(\xi) \leq C \tilde{P}(\xi) \quad \forall \xi \in \mathbb{R}^{n} .
$$

Remark 4.2. If $P(D)$ and $Q(D)$ are equally strong and hypoelliptic, then by Theorem 2.4 it follows that there are two constants $C, C^{\prime}>0$ such that

$$
\begin{array}{ll}
|P(\xi)|^{2} \leq C\left(1+|Q(\xi)|^{2}\right) & \forall \xi \in \mathbb{R}^{n} \\
|Q(\xi)|^{2} \leq C^{\prime}\left(1+|P(\xi)|^{2}\right) & \forall \xi \in \mathbb{R}^{n} .
\end{array}
$$

In particular, $\operatorname{deg} P=\operatorname{deg} Q$.

We recall from [13, Thm. 11.1.9] the following

Theorem 4.3. If $P(D)$ and $Q(D)$ are equally strong and $P(D)$ is hypoelliptic, then also $Q(D)$ is hypoelliptic. Moreover, if $d_{P}(\xi)$ and $d_{Q}(\xi)$ are the distance from $\xi \in \mathbb{R}^{n}$ to $V(P)$ and $V(Q)$ respectively, there exists then a constant $C>0$ such that

$$
C^{-1} \leq \frac{d_{P}(\xi)+1}{d_{Q}(\xi)+1} \leq C, \quad \forall \xi \in \mathbb{R}^{n} .
$$


Lemma 4.4. Let $P(D)$ and $Q(D)$ be two equally strong linear partial differential operators. Assume that $P$ (and hence $Q$ ) is hypoelliptic and of order $m$. Let $c_{P}$ and $c_{Q}$ be the constants defined in (2.2) for $P$ and $Q$ respectively. Then $c_{P}=c_{Q}$.

Proof. Let us first remark that condition (4.1) is equivalent to

$$
\tilde{C}^{-1} \leq \frac{d_{P}(\xi)}{d_{Q}(\xi)} \leq \tilde{C}, \quad|\xi| \gg 1
$$

for some $\tilde{C}>0$, because of condition (6) of Theorem 2.4.

Then (2.2) implies, for some $C, \bar{C}>0$,

$$
\begin{array}{lll}
|\xi|^{c_{P}} \leq C^{-1} d_{P}(\xi) \leq C^{-1} \tilde{C} d_{Q}(\xi) & \Rightarrow \quad c_{Q} \geq c_{P} \\
|\xi|^{c_{Q}} \leq \bar{C}^{-1} d_{Q}(\xi) \leq \bar{C}^{-1} \tilde{C} d_{P}(\xi) & \Rightarrow \quad c_{P} \geq c_{Q}
\end{array}
$$

and $c_{P}=c_{Q}$.

Remark 4.5. If $P(D)$ and $Q(D)$ are equally strong, hypoelliptic and of order $m$, and if $\gamma_{P}$ and $\gamma_{Q}$ are the constants defined in (2.5) for $P$ and $Q$ respectively, then (see Remark 2.5):

$$
m \leq \gamma_{P}, \gamma_{Q} \leq \frac{m}{c_{P}}=\frac{m}{c_{Q}} .
$$

Theorem 4.6. Let $P(D)$ and $Q(D)$ be two equally strong linear partial differential operators and let $\omega$ be a non-quasianalytic weight function. If $P$ is $*$-hypoelliptic then also $Q$ is $*$-hypoelliptic, for $*=(\omega)$ or $\{\omega\}$.

Proof. From Remark 2.10 we have that $P$ is hypoelliptic. Therefore also $Q$ is hypoelliptic, by Theorem 4.3 , and (4.2) is satisfied.

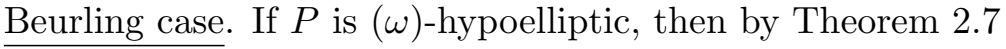

$$
\frac{\omega(\xi)}{d_{Q}(\xi)} \leq \tilde{C} \frac{\omega(\xi)}{d_{P}(\xi)} \longrightarrow 0 \quad \text { as }|\xi| \rightarrow+\infty
$$

and hence also $Q$ is $(\omega)$-hypoelliptic.

Roumieu case. If $P$ is $\{\omega\}$-hypoelliptic, then by Theorem 2.9

$$
\omega(\xi) \leq C d_{P}(\xi) \leq C \tilde{C} d_{Q}(\xi), \quad|\xi| \gg 1
$$

and hence also $Q$ is $\{\omega\}$-hypoelliptic.

Now, from Theorems 3.3 and 3.4, and Corollaries 3.14 and 3.15 it is easy to deduce the following:

Proposition 4.7. Let $P(D)$ and $Q(D)$ be two equally strong linear partial differential operators. Assume that $P$ (and hence $Q$ ) is hypoelliptic and of order $m$. Let $\omega$ be a non-quasianalytic weight function such that $\omega\left(t^{\gamma}\right)=o(\sigma(t))$, as $t \rightarrow+\infty$, where $\gamma:=\frac{m}{c_{P}}=\frac{m}{c_{Q}}$ as in (4.3) and $\sigma(t)=t^{1 / s}$ for some $s>1$. Let $\Omega$ be an open subset of $\mathbb{R}^{n}$ and $u \in \mathcal{D}^{\prime}(\Omega)$. Then

$$
\begin{aligned}
\mathcal{E}_{*}^{P}(\Omega) & =\mathcal{E}_{*}^{Q}(\Omega) \\
\mathrm{WF}_{*}^{P}(u) & =\mathrm{WF}_{*}^{Q}(u)
\end{aligned}
$$

for $*=(\omega)$ or $\{\omega\}$.

Let us now consider linear partial differential operators $P(x, D)$ with variable coefficients on an open subset $\Omega$ of $\mathbb{R}^{n}$. We recall from [13] the following:

Definition 4.8. A linear partial differential operator $P(x, D)$, with $C^{\infty}$ coefficients on an open subset $\Omega$ of $\mathbb{R}^{n}$, is said to have constant strength in $\Omega$ if, for every $x_{0}, y_{0} \in \Omega$, the differential operators with constant coefficients $P\left(x_{0}, D\right)$ and $P\left(y_{0}, D\right)$ are equally strong.

The following result is due to Hörmander and Taylor (cf. Theorems 13.4.1, 13.4.2 and 13.4.4 of [13]): 
Theorem 4.9. Let $\Omega$ be an open subset of $\mathbb{R}^{n}$ and $P(x, D)$ a linear partial differential operator with coefficients in $C^{\infty}(\Omega)$. Assume that $P(x, D)$ has constant strength. Then the following conditions are equivalent:

(1) $P(x, D)$ is hypoelliptic in $\Omega$, i.e.

$$
\text { sing supp } u=\operatorname{sing} \operatorname{supp} P(\cdot, D) u, \quad u \in \mathcal{D}^{\prime}(\Omega) ;
$$

(2) $P\left(x_{0}, D\right)$ is hypoelliptic for some $x_{0} \in \Omega$;

(3) $P\left(x_{0}, D\right)$ is hypoelliptic for all $x_{0} \in \Omega$.

Moreover, if one of the above equivalent conditions is satisfied, then $P(x, D)$ is micro-hypoelliptic in $\Omega$, i.e.

$$
\mathrm{WF}(u)=\mathrm{WF}(P(\cdot, D) u), \quad u \in \mathcal{D}^{\prime}(\Omega) .
$$

In the case of $\omega$-hypoellipticity, from Theorem 4.6 we immediately obtain that for a linear partial differential operator with $C^{\infty}$ coefficients $P(x, D)$ with constant strength, $P\left(x_{0}, D\right)$ is $*$-hypoelliptic for some $x_{0} \in \Omega$ if and only if $P\left(x_{0}, D\right)$ is $*$-hypoelliptic for all $x_{0} \in \Omega$, where $*=(\omega)$ or $\{\omega\}$.

As a consequence of Theorem 4.9 and Lemma 4.4 we have that if $P(x, D)$ is a hypoelliptic linear partial differential operator of order $m$ with $C^{\infty}$ coefficients and of constant strength in an open subset $\Omega$ of $\mathbb{R}^{n}$, there is then a unique constant $c_{P} \in(0,1] \cap \mathbb{Q}$ satisfying $(2.2)$, in the sense that $c_{P\left(x_{0}, D\right)}=c_{P\left(x_{1}, D\right)}=: c_{P}$ for all $x_{0}, x_{1} \in \Omega$. We can then uniquely define

$$
\gamma_{P}:=\frac{m}{c_{P}} \text {. }
$$

Corollary 4.10. Let $\Omega$ be an open subset of $\mathbb{R}^{n}$ and $u \in \mathcal{D}^{\prime}(\Omega)$. Let $P(x, D)$ be a linear partial differential operator with coefficients in $C^{\infty}(\Omega)$. Assume that $P(x, D)$ has constant strength in $\Omega$ and that $P\left(x_{0}, D\right)$ is hypoelliptic for some $x_{0} \in \Omega$. Let $\omega$ be a non-quasianalytic weight function such that $\omega\left(t^{\gamma}\right)=o(\sigma(t))$, as $t \rightarrow+\infty$, where $\gamma=\gamma_{P}$ is the constant defined in (4.6) and $\sigma(t)=t^{1 / s}$ for some $s>1$. Then

$$
\begin{aligned}
\mathcal{E}_{*}^{P(x, D)}(\Omega) & =\mathcal{E}_{*}^{P\left(x^{\prime}, D\right)}(\Omega) & \forall x, x^{\prime} \in \Omega \\
\mathrm{WF}_{*}^{P(x, D)}(u) & =\mathrm{WF}_{*}^{P\left(x^{\prime}, D\right)}(u) & \forall x, x^{\prime} \in \Omega
\end{aligned}
$$

for $*=(\omega)$ or $\{\omega\}$.

Proof. It follows from Theorem 4.9 and Proposition 4.7.

Corollary 4.11. Let $\Omega$ be an open subset of $\mathbb{R}^{n}$ and $u \in \mathcal{D}^{\prime}(\Omega)$. Let $P(x, D)$ be a linear partial differential operator with coefficients in $C^{\infty}(\Omega)$. Assume that $P(x, D)$ has constant strength in $\Omega$ and that $P\left(x_{0}, D\right)$ is hypoelliptic for some $x_{0} \in \Omega$. Let $\omega$ be a non-quasianalytic weight function such that $\omega\left(t^{\gamma}\right)=o(\sigma(t))$, as $t \rightarrow+\infty$, where $\gamma=\gamma_{P}$ is the constant defined in (4.6) and $\sigma(t)=t^{1 / s}$ for some $s>1$. Then

$$
\mathrm{WF}_{*}(u) \subseteq \mathrm{WF}_{*}^{P\left(x_{1}, D\right)}(u) \cup\left(\bigcap_{x^{\prime} \in \Omega} \Sigma_{x^{\prime}}\right) \quad \forall x_{1} \in \Omega,
$$

where $*=(\omega)$ or $\{\omega\}$ and

$$
\Sigma_{x^{\prime}}:=\left\{(x, \xi) \in \Omega \times\left(\mathbb{R}^{n} \backslash\{0\}\right): P_{m\left(x^{\prime}\right)}\left(x^{\prime}, \xi\right)=0\right\}
$$

with $P_{m\left(x^{\prime}\right)}\left(x^{\prime}, \xi\right)$ the principal part of $P\left(x^{\prime}, \xi\right)$.

Proof. By Theorem 13 of [3] we have that

$$
\begin{aligned}
\mathrm{WF}_{*}(u) & \subseteq \mathrm{WF}_{*}^{P\left(x^{\prime}, D\right)}(u) \cup \Sigma_{x^{\prime}} & & \forall x^{\prime} \in \Omega \\
& =\mathrm{WF}_{*}^{P\left(x_{1}, D\right)}(u) \cup \Sigma_{x^{\prime}} & & \forall x_{1}, x^{\prime} \in \Omega
\end{aligned}
$$

by Corollary 4.10 . This proves the thesis. 
Corollary 4.12. Let $\Omega$ be an open subset of $\mathbb{R}^{n}$ and $u \in \mathcal{D}^{\prime}(\Omega)$. Let $P(x, D)$ be a linear partial differential operator with coefficients in $C^{\infty}(\Omega)$. Assume that $P(x, D)$ has constant strength in $\Omega$ and that $P\left(x_{0}, D\right)$ is elliptic for some $x_{0} \in \Omega$. Let $\omega$ be a non-quasianalytic weight function such that $\omega\left(t^{\gamma}\right)=o(\sigma(t))$, as $t \rightarrow+\infty$, where $\gamma=\gamma_{P}$ is the constant defined in (4.6) and $\sigma(t)=t^{1 / s}$ for some $s>1$.

Then $P\left(x_{1}, D\right)$ is *-micro-hypoelliptic for all $x_{1} \in \Omega$, i.e.

$$
\mathrm{WF}_{*}(u)=\mathrm{WF}_{*}\left(P\left(x_{1}, D\right) u\right) \quad \forall x_{1} \in \Omega,
$$

for $*=(\omega)$ or $\{\omega\}$.

Proof. By [3, Rem. 14] we have that

$$
\mathrm{WF}_{*}(u)=\mathrm{WF}_{*}^{P\left(x_{0}, D\right)}(u)
$$

by the ellipticity of $P\left(x_{0}, D\right)$.

Therefore, by Corollary 4.10 and [3, Rem. 12 and Prop. 9], for all $x_{1} \in \Omega$ :

$$
\begin{aligned}
\mathrm{WF}_{*}(u) & =\mathrm{WF}_{*}^{P\left(x_{0}, D\right)}(u)=\mathrm{WF}_{*}^{P\left(x_{1}, D\right)}(u) \\
& =\mathrm{WF}_{*}^{P\left(x_{1}, D\right)}\left(P\left(x_{1}, D\right) u\right) \subseteq \mathrm{WF}_{*}\left(P\left(x_{1}, D\right) u\right) \subseteq \mathrm{WF}_{*}(u),
\end{aligned}
$$

and hence the thesis.

The following corollary can be proved directly with the results obtained in [9], but we present it here as a consequence of the previous theorems.

Corollary 4.13. Let $\Omega$ be an open subset of $\mathbb{R}^{n}$ and $u \in \mathcal{D}^{\prime}(\Omega)$. Let $P(x, D)$ be a linear partial differential operator with coefficients in $\mathcal{E}_{\{\sigma\}}(\Omega)$. Assume that $P(x, D)$ has constant strength in $\Omega$ and that $P\left(x_{0}, D\right)$ is elliptic for some $x_{0} \in \Omega$. Let $\omega$ be a non-quasianalytic weight function such that $\omega\left(t^{\gamma}\right)=o\left(t^{1 / s}\right)$, for $\gamma=\gamma_{P}$ as in (4.6) and $s>1$, and such that $\omega(t)=o(\sigma(t))$ for a non-quasianalytic weight function $\sigma$ satisfying, for some $c>0$,

$$
\int_{1}^{+\infty} \frac{\sigma(t y)}{t^{2}} d t \leq c \sigma(t)+c \quad y>0 .
$$

Then

$$
\mathrm{WF}_{*}(u)=\mathrm{WF}_{*}(P(\cdot, D) u)=\mathrm{WF}_{*}\left(P\left(x_{1}, D\right) u\right) \quad \forall x_{1} \in \Omega,
$$

for $*=(\omega)$ or $\{\omega\}$.

Proof. By Theorem 3 of [9] we have that

$$
\mathrm{WF}_{*}(u)=\mathrm{WF}_{*}(P(\cdot, D) u) .
$$

By Corollary 4.12 we have

$$
\mathrm{WF}_{*}(u)=\mathrm{WF}_{*}\left(P\left(x_{1}, D\right) u\right) \quad \forall x_{1} \in \Omega .
$$

Therefore also

$$
\mathrm{WF}_{*}(P(\cdot, D) u)=\mathrm{WF}_{*}\left(P\left(x_{1}, D\right) u\right) \quad \forall x_{1} \in \Omega
$$

is valid.

Example 4.14. Let $q(\xi) \geq 0$ be a hypoelliptic polynomial, $h, m, m^{\prime} \in \mathbb{N}_{0}, m>m^{\prime}$, and consider the operator $P(x, D)$ with symbol

$$
p(x, \xi):=|x|^{2 h} q(\xi)^{m}+q(\xi)^{m^{\prime}} .
$$

It was proved in [8, Ex. 3.6] that there exist $0 \leq \delta<\rho \leq 1$ such that, for $a<d:=\rho-\delta<1$, $a / d<r<1, \sigma(t)=t^{r}$ and $\omega(t)=o\left(t^{a}\right)$ :

$$
|p(x, \xi)| \geq c e^{-\omega(\xi)} \quad \forall x \in \mathbb{R}^{n},|\xi| \gg 1
$$


for some $c>0$, and

$$
\left|D_{x}^{\alpha} D_{\xi}^{\beta} p(x, \xi)\right| \leq C^{|\alpha|+|\beta|} \beta ! e^{\frac{1}{k} \varphi_{\sigma}^{*}(|\alpha| k)}|p(x, \xi)|(1+|\xi|)^{-\rho|\beta|+\delta|\alpha|} \quad \forall x \in \mathbb{R}^{n},|\xi| \gg 1
$$

for some $C>0$ and $k \in \mathbb{N}$.

By $[8$, Thm. 3.4] and [9, Thm. 2] we have

$$
\mathrm{WF}_{(\omega)}(u)=\mathrm{WF}_{(\omega)}(P(\cdot, D) u), \quad u \in \mathcal{E}_{(\omega)}^{\prime}(\Omega) .
$$

Analogously, since (4.9) and (4.10) hold also for every fixed $x_{1} \in \Omega$, we have that

$$
\mathrm{WF}_{(\omega)}(u)=\mathrm{WF}_{(\omega)}\left(P\left(x_{1}, D\right) u\right), \quad \forall x_{1} \in \Omega,
$$

and hence (4.8) holds for all $u \in \mathcal{E}_{(\omega)}^{\prime}(\Omega)$, and hence for all $u \in \mathcal{E}^{\prime}(\Omega)$.

Note that $P$ is not of constant strength, and that $P$ is elliptic in some $x_{0} \in \Omega$ if and only if $q(\xi)$ is elliptic.

Example 4.15. Let us consider, for $m \geq 1$, the operator

$$
P\left(x, y, D_{x}, D_{y}\right)=D_{x}^{2 m}+x^{2 h} D_{y}^{2 m+2} .
$$

It was proved in [24, Thm. 3.1] that if $h>m$ and $\omega(t)=t^{1 / s}$, with $s \geq 1+\frac{1}{m}$, then

$$
\mathrm{WF}_{\{\omega\}}(u)=\mathrm{WF}_{\{\omega\}}(P(\cdot, D) u), \quad \forall u \in \mathcal{D}^{\prime}\left(\mathbb{R}^{2}\right) .
$$

However, for fixed $\left(x_{1}, y_{1}\right)=\left(0, y_{1}\right)$ the operator $P\left(0, y_{1}, D_{x}, D_{y}\right)=D_{x}^{2 m}$ is not $\{\omega\}$-hypoelliptic, since every $f(x, y)=f(y) \in \mathcal{D}^{\prime}(\mathbb{R}) \backslash \mathcal{E}_{\{\omega\}}(\mathbb{R})$ solves $P\left(0, y_{1}, D_{x}, D_{y}\right) f=0$, and hence it is not $\{\omega\}$-microhypoelliptic by [3, Cor. 11]:

$$
\mathrm{WF}_{\{\omega\}}\left(P\left(0, y_{1}, D_{x}, D_{y}\right) f\right) \nsubseteq \mathrm{WF}_{\{\omega\}}(f) .
$$

In particular, by (4.11):

$$
\mathrm{WF}_{\{\omega\}}\left(P\left(0, y_{1}, D_{x}, D_{y}\right) f\right) \nsubseteq \mathrm{WF}_{\{\omega\}}(P(\cdot, D) f) .
$$
$\mathbb{R}^{2}$.

Note that $P$ does not have constant strength and $P\left(x_{0}, y_{0}, D_{x}, D_{y}\right)$ is not elliptic for any $\left(x_{0}, y_{0}\right) \in$

Acknowledgements: The authors were partially supported by FAR2011 (Università di Ferrara) and "Fondi per le necessità di base della ricerca" 2012 and 2013 (Università di Ferrara). The first author is member of the Gruppo Nazionale per l'Analisi Matematica, la Probabilità e le loro Applicazioni (GNAMPA) of the Instituto Nazionale di Alta Matematica (INdAM). The research of the second author was partially supported by MINECO of Spain, Project MTM2013-43540-P.

\section{References}

[1] A.A. Albanese, D. Jornet, A. Oliaro, Quasianalytic wave front sets for solutions of linear partial differential operators, Integr. Equ. Oper. Theory 66 (2010), 153-181.

[2] C. Boiti, D. Jornet, The problem of iterates in some classes of ultradifferentiable functions, to appear in "Operator Theory: Advances and Applications", Birkhauser, Basel.

[3] C. Boiti, D. Jornet, J. Juan-Huguet, Wave front set with respect to the iterates of an operator with constant coefficients, Abstr. Appl. Anal. 2014, Article ID 438716 (2014), pp. 1-17, http://dx.doi.org/10.1155/2014/438716

[4] P. Bolley, J. Camus, C. Mattera, Analyticité microlocale et itérés d'operateurs hypoelliptiques, Séminaire GoulaouicSchwartz, 1978-79, Exp N.13, École Polytech., Palaiseau.

[5] J. Bonet, C. Fernández, R. Meise, Characterization of the $\omega$-hypoelliptic convolution operators on ultradistributions, Ann. Acad. Sci. Fenn. Math. 25 (2000), 261-284

[6] J. Bonet, R. Meise, S.N. Melikhov, A comparison of two different ways of define classes of ultradifferentiable functions, Bull. Belg. Math. Soc. Simon Stevin 14 (2007), 425-444.

[7] R.W. Braun, R. Meise, B.A. Taylor, Ultradifferentiable functions and Fourier analysis, Result. Math. 17 (1990), 206-237.

[8] C. Fernández, A. Galbis, D. Jornet, w-hypoelliptic differential operators of constant strength, J. Math. Anal. Appl. 297 (2004), 561-576. 
[9] C. Fernández, A. Galbis, D. Jornet, Pseudodifferential operators of Beurling type and the wave front set, J. Math. Anal. Appl. 340 (2008), 1153-1170.

[10] L. Hörmander, On Interior Regularity of the Solutions of Partial Differential Equations, Comm. Pure Appl. Math. Vol. XI (1958), 197-218.

[11] L. Hörmander, Uniqueness theorems and wave front sets for solutions of linear partial differential equations with analytic coefficients, Comm. Pure Appl. Math. 24 (1971), 671-704.

[12] L. Hörmander, The Analysis of Linear Partial Differential Operators I, Springer-Verlag, Berlin (1990).

[13] L. Hörmander, The Analysis of Linear Partial Differential Operators II, Springer-Verlag, Berlin (1983).

[14] J. Juan-Huguet, Iterates and Hypoellipticity of Partial Differential Operators on Non-Quasianalytic Classes, Integr. Equ. Oper. Theory 68 (2010), 263-286.

[15] J. Juan-Huguet, A Paley-Wiener type theorem for generalized non-quasianalytic classes, Studia Math. 208, n.1 (2012), 31-46.

[16] H. Komatsu, A characterization of real analytic functions, Proc. Japan Acad. 36 (1960), 90-93.

[17] T. Kotake, M.S. Narasimhan, Regularity theorems for fractional powers of a linear elliptic operator, Bull. Soc. Math. France 90 (1962), 449-471.

[18] M. Langenbruch, P-Funktionale und Randwerte zu hypoelliptischen Differentialoperatoren, Math. Ann. 239(1) (1979), 55-74.

[19] M. Langenbruch, Fortsetzung von Randwerten zu hypoelliptischen Differentialoperatoren und partielle Differentialgleichungen, J. Reine Angew. Math. 311/312 (1979), 57-79.

[20] M. Langenbruch, On the functional dimension of solution spaces of hypoelliptic partial differential operators, Math. Ann. 272 (1985), 217-229.

[21] M. Langenbruch, Bases in solution sheaves of systems of partial differential equations, J. Reine Angew. Math. 373 (1987), 1-36.

[22] G. Métivier, Propriété des itérés et ellipticité, Comm. Partial Differential Equations 3 (9) (1978), 827-876.

[23] E. Newberger, Z. Zielezny, The growth of hypoelliptic polynomials and Gevrey classes, Proc. Amer. Math. Soc. 39, n. 3 (1973), 547-552.

[24] L. Rodino, On the problem of the hypoellipticity of the linear partial differential equations, Developments in Partial Differential Equations and Applications to Mathematical Physics, Edited by G. Buttazzo, Plenum Press, New York, 1992.

[25] L. Rodino, Linear partial differential operators in Gevrey spaces, World Scientific, 1993.

[26] L. Zanghirati, Iterates of a class of hypoelliptic operators and generalized Gevrey classes, Boll. U.M.I. Suppl. 1 (1980), 177-195.

Dipartimento di Matematica e Informatica, Università di Ferrara, Via Machiavelli n. 30, 44121 FerRARA, ITALY

E-mail address: chiara.boiti@unife.it

Instituto Universitario de Matemática Pura y Aplicada IUMPA, Universitat Politècnica de València, C/Camino de Vera, s/n, E-46071 Valencia, Spain

E-mail address: djornet@mat.upv.es 